\title{
An angle-based subspace anomaly detection approach to high-dimensional data: With an application to industrial fault detection
}

\author{
Liangwei Zhang*, Jing Lin, Ramin Karim \\ Division of Operation and Maintenance Engineering, Luleå University of Technology, 97187 Luleå, Sweden
}

\section{A R T I C L E I N F O}

\section{Article history:}

Received 11 February 2015

Received in revised form

17 April 2015

Accepted 30 May 2015

Available online 11 June 2015

Keywords:

Big data analytics

Anomaly detection

High-dimensional data

Fault detection

\begin{abstract}
A B S T R A C T
The accuracy of traditional anomaly detection techniques implemented on full-dimensional spaces degrades significantly as dimensionality increases, thereby hampering many real-world applications. This work proposes an approach to selecting meaningful feature subspace and conducting anomaly detection in the corresponding subspace projection. The aim is to maintain the detection accuracy in high-dimensional circumstances. The suggested approach assesses the angle between all pairs of two lines for one specific anomaly candidate: the first line is connected by the relevant data point and the center of its adjacent points; the other line is one of the axis-parallel lines. Those dimensions which have a relatively small angle with the first line are then chosen to constitute the axis-parallel subspace for the candidate. Next, a normalized Mahalanobis distance is introduced to measure the local outlier-ness of an object in the subspace projection. To comprehensively compare the proposed algorithm with several existing anomaly detection techniques, we constructed artificial datasets with various high-dimensional settings and found the algorithm displayed superior accuracy. A further experiment on an industrial dataset demonstrated the applicability of the proposed algorithm in fault detection tasks and highlighted another of its merits, namely, to provide preliminary interpretation of abnormality through feature ordering in relevant subspaces.
\end{abstract}

(c) 2015 Elsevier Ltd. All rights reserved.

\section{Introduction}

Increasing attention is being devoted to Big Data Analytics and its attempt to extract information, knowledge and wisdom from Big Data. In the literature, the concept of Big Data is mainly characterized by the three "Vs" (Volume, Velocity and Variety) [1] together with " $\mathrm{c}$ " to denote "complexity" [2]. High dimensionality, one measure of the volume of data (the other measure being instance size) [3], presents a challenge to Big Data Analytics in industry. For example, high dimensionality has been recognized as the distinguishing feature of modern field reliability data (incl. System Operating/Environmental

\footnotetext{
Abbreviations: ABOD, Angle-Based Outlier Detection; ABSAD, Angle-Based Subspace Anomaly Detection; ANN, Artificial Neuron Network; AUC, Area Under Curve; CMMS, Computerized Maintenance Management System; FCM, Fuzzy C-Means; FPR, False Positive Rate; ICA, Independent Component Analysis; LOF, Local Outlier Factor; MSPC, Multivariate Statistical Process Control; PCA, Principal Component Analysis; ROC, Receiver Operating Characteristic; SNN, Shared Nearest Neighbors; SOD, Subspace Outlier Detection; SOE, System Operating/Environmental (data); SPE, Squared Prediction Error; SVM, Support Vector Machine; TPR, True Positive Rate

* Corresponding author.

E-mail address: liangwei.zhang@ltu.se (L. Zhang).
}

data, or SOE data), i.e. periodically generated large vectors of dynamic covariate values [4]. Due to the "curse of dimensionality", it has also been regarded as the primary complexity of multivariate analysis and covariate-response analysis in reliability applications $[5,6]$.

Anomaly detection, also called outlier detection, aims to detect observations which deviate so much from others that they are suspected of being generated by different mechanisms [7]. Efficient detection of such outliers can help, in a timely way, to rectify faulty behavior of a system and, consequently, to avoid losses. In view of this, anomaly detection techniques have been applied to various fields, including industrial fault detection, network intrusion detection and so forth [8-10]. High dimensionality complicates anomaly detection tasks because the degree of data abnormality in relevant dimensions can be obscured or even masked by irrelevant dimensions [5,11,12]. For instance, in an industrial case (see Section 4.2), when detecting the fault "cavitation" in a hydro-turbine, many irrelevant dimensions (e.g. "hydraulic oil level" and "output power") can easily conceal signals relevant to this anomaly (e.g. "substructure vibration") and impede the discovery of the fault. Moreover, outliers are very similar to normal objects in high-dimensional spaces from the perspective of both probability and distance [5]. The use of 


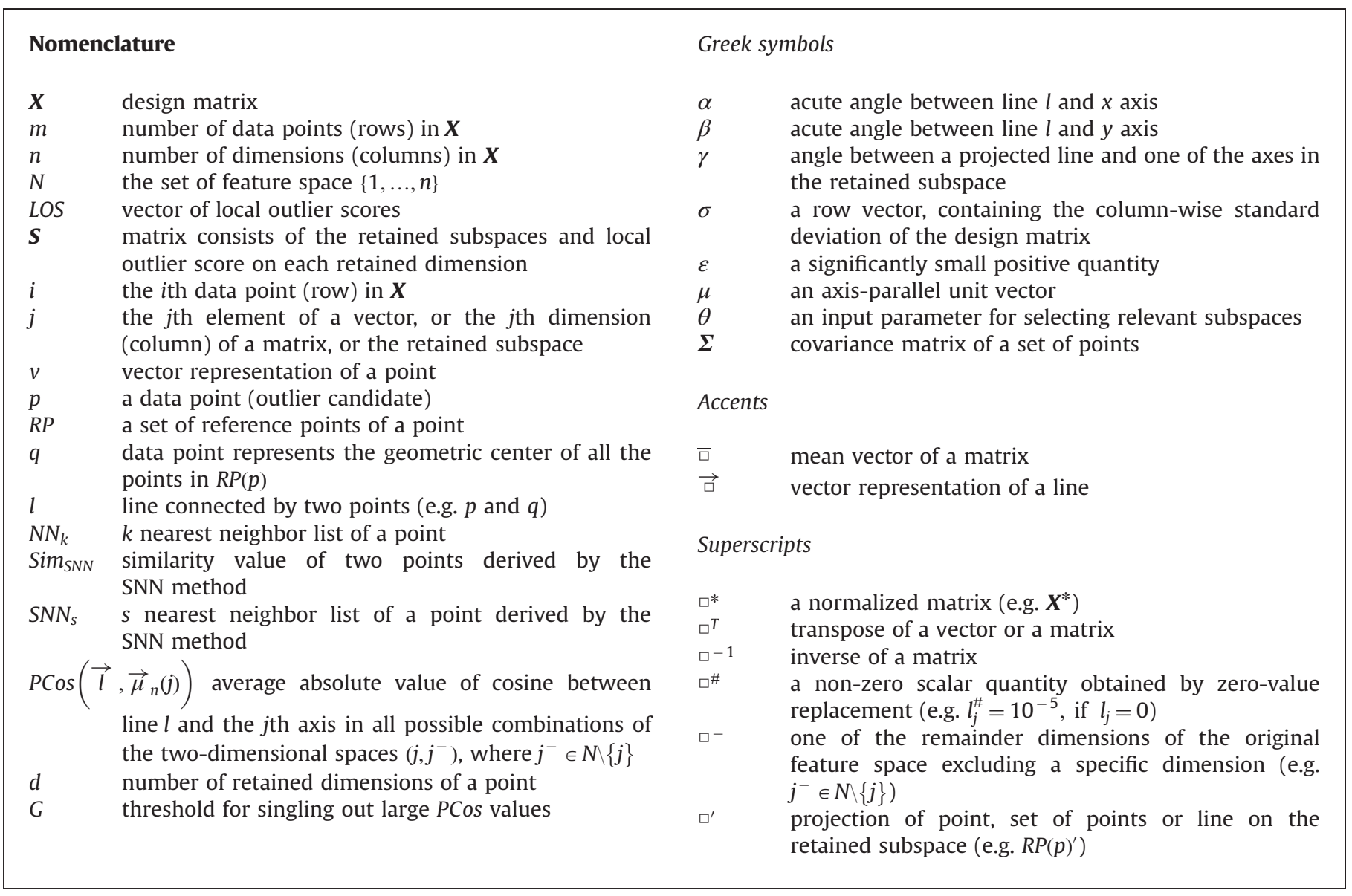

The symbol $\square$ denotes a placeholder.

traditional techniques to conduct anomaly detection in fulldimensional spaces is problematic, as anomalies normally appear in a small subset of all the dimensions.

Industrial fault detection aims to identify defective states of a process in complex industrial systems, subsystems and components. Early discovery of system faults may ensure the reliability and safety of industrial systems and reduce the risk of unplanned breakdown $[13,14]$. Fault detection is a vital component of an Integrated Systems Health Management system; it has been considered as one of the most promising applications wherein reliability meets Big Data [4]. From the data processing point of view, methods of fault detection can be classified into three categories: (i) model-based, online, datadriven methods; (ii) signal-based methods; and (iii) knowledgebased, history data-driven methods [13]. Given the complexity of modern systems, it is too complicated to explicitly represent the real process with models or to define the signal patterns of the system process. Thus, knowledge-based fault detection methods, which intend to acquire underlying knowledge from large amounts of empirical data, are more desirable than other methods [13]. Existing knowledge-based fault detection methods can be further divided into supervised and unsupervised ones, depending on whether the raw data have been labeled or not, i.e. indicating whether the states of the system process in historical data are normal or faulty. Generally, supervised learning methods like Support Vector Machine (SVM), Fuzzy C-Means (FCM), Artificial Neural Network (ANN), and several others can provide reasonably accurate results in detecting or even isolating the hidden faults $[9,15]$. However, when there is a lack of sufficient labeled data, often the case in reality, fault detection must resort to unsupervised methods. In unsupervised fault detection methods, normal operating conditions are modeled beforehand, and faults are detected as deviations from the normal behavior. A variety of unsupervised learning algorithms have been adopted for this purpose, such as Deep Belief Network, $k$ Nearest Neighbors, and other clustering-based methods [16,17], but few have tackled the challenges of high-dimensional datasets.

Other types of Multivariate Statistical Process Control (MSPC) methods, including Principle Component Analysis (PCA) and Independent Component Analysis (ICA), have also been widely used in fault detection $[18,19]$. But PCA-based models assume multivariate normality of the in-control data, while ICA-based models assume latent variables are non-Gaussian distributed [20,21]. Both MSPC methods make strong assumptions about the specific data distributions, thereby limiting their performance in real-world applications [22]. Moreover, although PCA and ICA can reduce dimensions and extract information from highdimensional datasets, their original purpose was not to detect anomalies. Further research has confirmed PCA-based models are not sensitive to faults occurring on the component level [23]. To improve this, several studies have integrated MSPC methods with assumption-free techniques, such as the density-based Local Outlier Factor (LOF) approach [22,24]. Though better accuracy has been reported, LOF still suffers from the "curse of dimensionality", i.e. the accuracy of LOF implemented on full-dimensional spaces degrades as dimensionality increases, as will be shown in Section 4.1.

Although in many industrial applications for fault detection, detecting anomalies from high-dimensional data remains relatively under-explored, several theoretical studies (see Section 2 for a review) have started to probe this issue, including, for example, subspace anomaly detection by random projection or heuristic searches over subspaces. These methods, however, are either arbitrary in selecting subspaces or computationally intensive. 
To deal with the aforementioned challenges, this paper proposes an approach to selecting meaningful feature subspace and conducting anomaly detection in the corresponding subspace projection. The aim is to maintain the detection accuracy in high-dimensional circumstances. The suggested approach assesses the angle between all pairs of two lines for one anomaly candidate: the first line is connected by the concerned data point and the center of its adjacent points; the other is one of the axis-parallel lines. The dimensions, which have a relatively small angle with the first line, are then chosen to constitute the axis-parallel subspace of the anomaly candidate. Next, a normalized Mahalanobis distance is introduced to measure the local outlier-ness of the data point in the subspace projection and a consolidated algorithm integrating the above steps is proposed. The algorithm yields an outlier score for a specific data instance and also outputs a feature ordering list indicating the degree of deviation at each individual dimension. This feature ordering in relevant subspaces can be leveraged to give a preliminary explanation for data abnormality. A comprehensive evaluation of the algorithm is investigated through synthetic datasets and an industrial fault detection dataset. The reasons for using synthetic datasets are the following: (i) to compare the suggested algorithm with other alternatives and examine their performance under various dimensionality settings, the dimensionality of the dataset should be adjustable; (ii) to verify whether the proposed algorithm can select meaningful subspace on which anomalies deviate significantly from their neighboring points, the exact position of anomaly-relevant attributes need to be known in advance. Neither requirement can be easily met by real-world datasets.

The rest of this paper proceeds as follows. In Section 2, we briefly review existing anomaly detection techniques, especially subspace anomaly detection approaches, and discuss the challenge posed by high dimensionality. In Section 3, we illustrate the Angle-Based Subspace Anomaly Detection (ABSAD) algorithm, especially the process of selecting relevant subspace, in detail. The proposed algorithm is evaluated on both synthetic datasets and an industrial fault detection dataset in Section 4; we also compare our algorithm with other alternatives. Finally, Section 5 concludes the work.

\section{Overview of anomaly detection techniques}

In this section, we firstly introduce the taxonomy of anomaly detection techniques and confine the scope of this study. Afterwards, we review related literature and find the gap, and then elucidate the main motivation of this paper.

\subsection{Taxonomy of anomaly detection techniques}

A few surveys regarding anomaly detection have been conducted: some of them reviewed different types of anomaly detection techniques [25]; some focused on applications in different domains [26]; while others were targeted at solving special problems (e.g. high-dimensional data) [12]. According to these surveys, anomaly detection techniques can be roughly classified into different categories, as shown in Fig. 1, including: supervised versus unsupervised, depending on whether the raw data are labeled or not; global versus local, depending on the size of the reference set; and full-space versus subspace, depending on the number of considered attributes when defining anomalies. On the other hand, corresponding to the theoretical origin, anomaly detection techniques can be divided into statistical, classification-based, nearest-neighbor-based, clusteringbased, information theoretical, spectral models, and so on.

In this paper, we consider unsupervised subspace anomaly detection for high-dimensional continuous data in a local sense. The reason of selecting this combination of models is explained as follows.

\section{1) Supervised versus unsupervised}

In an ordinary binary classification problem, supervised algorithms need plentiful positive (abnormal) and negative (normal) data to learn the underlying generating mechanisms of different classes of data. However, for most anomaly detection applications, abnormal data are generally insufficient [23]. This problem becomes worse as dimensionality increases. In order to show this, we take the same example given in [5] which states that even a huge training set of a trillion examples only covers a fraction of $10^{-18}$ of a moderate 100 dimensional input space. In addition, though supervised algorithms typically have high accuracy in detecting anomalies that have occurred before, it is not good at detecting anomalies that have never happened before.

2) Global versus local

Global and local anomaly detection models differ in the scope of reference objects which one particular point may deviate from. In the former case, the reference objects are the whole dataset (e.g. the angle-based outlier detection technique), while in the latter case (e.g. $k$ nearest neighbors) subsets of all the data instances are taken into account $[27,28]$. A formal definition of a local outlier was given in [29] and the problems of evaluating the outlier-ness of a point from a global view were also discussed in that paper. For many real-world datasets, which have a complex structure, data are generated by various mechanisms. This is especially true for high-dimensional datasets in which a certain generating mechanism can normally affect only a subset of all the attributes. Under such circumstances, local outlier detection techniques are usually preferred over global ones in terms of accuracy [28].

3) Full-space versus subspace

In high-dimensional spaces, the degree of deviation in some attributes may be obscured or covered by other irrelevant attributes $[5,11,12]$. To explain this, we look at a toy example as follows. In Fig. 2(a), randomly generated samples are plotted in a three-dimensional coordinate system. An outlier is placed in the dataset and marked as a red cross. The outlier behaves normally in the axis $x$ and $y$ as indicated in Fig. 2(b) but deviates significantly from other points in the $z$ axis as shown in Fig. 2(c). From the perspective of distance, the fact that the outlier lies close to the cluster center in the $x$ and $y$ dimensions compensates for the deviation of the outlier from the center of the $z$ dimension. On the other hand, from the perspective of probability, the high likelihood of the value occurrence of the outlier in the $x$ and $y$ dimensions counteracts the low probability of abnormal value occurrence in the $z$ axis to some extent Consequently, neither distance-based approaches nor statistical models can effectively detect the severity of abnormality in the relevant subspace, namely the $z$ dimension in this example. This effect of sunken abnormality becomes more severe as the number of irrelevant dimensions increases. As identified in $[11,12]$, when the ratio of relevant and irrelevant attributes is high, traditional outlier detection techniques can still work even in a very high-dimensional setting. However, a low ratio of relevant and irrelevant attributes may greatly impede the separability of different data-generating mechanisms, and hence lead to the deterioration of traditional anomaly detection techniques implemented on full-dimensional spaces. In light of this consideration, researchers have started to probe into subspace anomaly detection techniques recently.

\subsection{Model analysis of anomaly detection}

A large portion of unsupervised anomaly detection techniques are distance-based or density-based [20]. An example of the distance-based models is the algorithm $D B(p, d)$. In that algorithm an object is claimed to be an outlier if there are at least $p$ percentage 


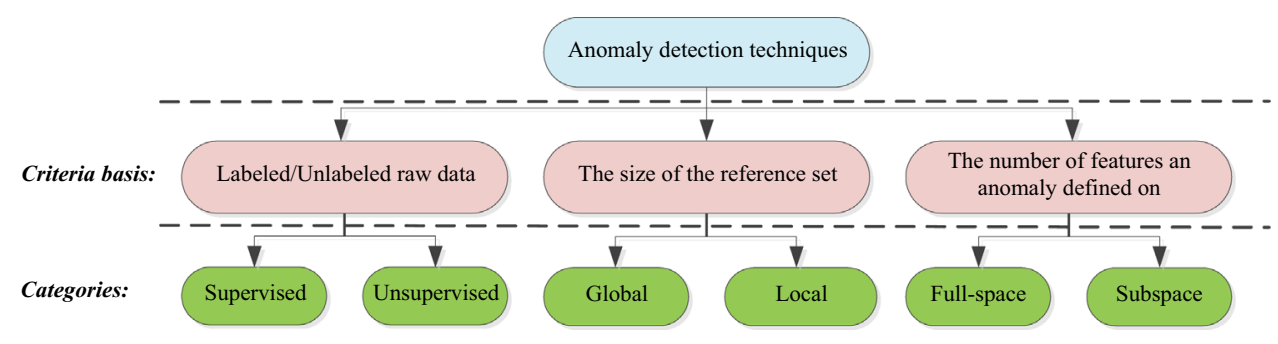

Fig. 1. Taxonomy of anomaly detection techniques.

b
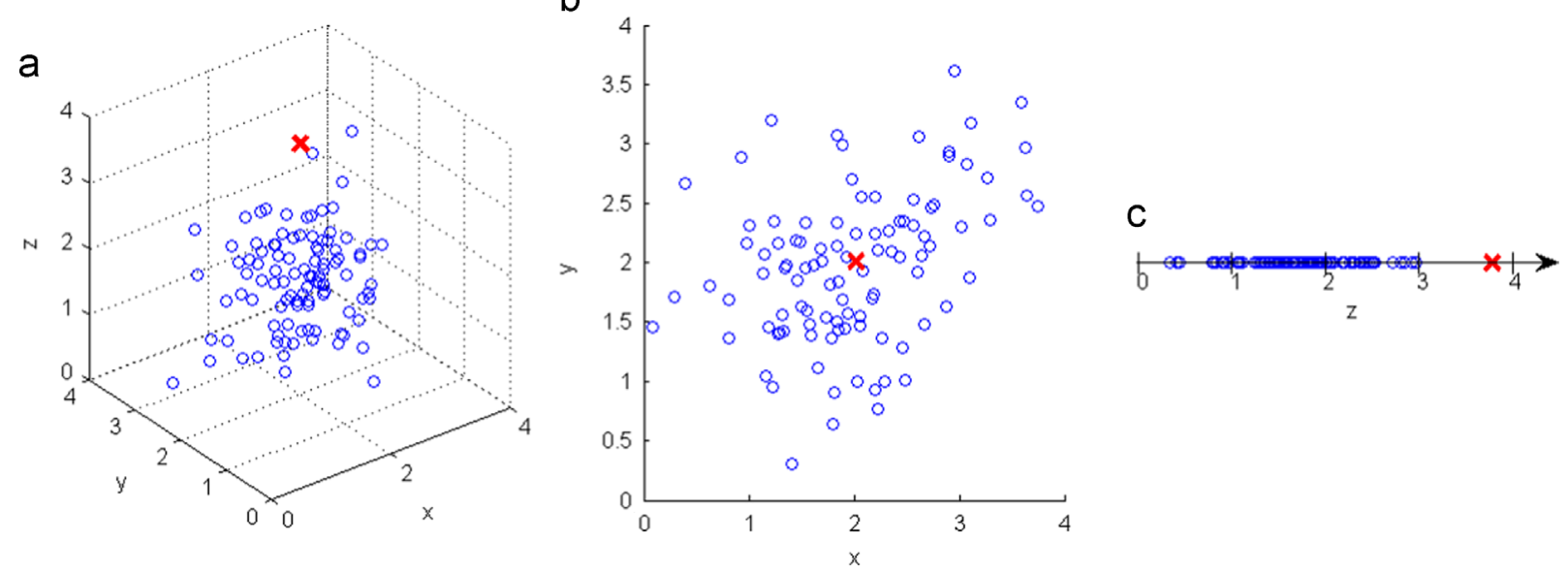

Fig. 2. Irrelevant attributes $x$ and $y$ conceal the deviation in relevant dimension $z$.

of other points in the dataset which have distance greater than $d$ from the object [27]. However, distanced-based approaches cannot effectively detect outliers from datasets with various densities [29]. Thus, another type of approach measuring local density of points was proposed. One of the best-known and most popular densitybased approaches is Local Outlier Factor (LOF). The LOF approach computes the average ratio of the local reachability density of a point and those of the point's nearest neighbors [29]. However, in a broad range of data distributions, distances between pairwise data points concentrate to a certain level as dimensionality increases, i.e. the distance-based nearest neighbor approaches to the farthest neighbor [30]. The loss of contrast in distance measurement leads to the concept of proximity and neighborhood in high-dimensional spaces becoming less meaningful [30], which undermines the theoretical basis that most of the distance-based and densitybased anomaly detection approaches rely on. In addition, for the type of local outlier detection models, it is difficult to define an appropriate reference set that can precisely reflect the locality of an object in high-dimensional spaces.

To alleviate the drawbacks of distance-based models in highdimensional spaces, a relatively stable metric in high-dimensional spaces - angle - was used in anomaly detection [28], [31]. The AngleBased Outlier Detection (ABOD) approach measures the variance in the angles between the difference vectors of a data point to the other points. Normal objects lying inside a cluster always have a large value of such variance, whereas outliers typically have very small variance in the angles. Even though the authors claimed that ABOD can alleviate the effect of the "curse of dimensionality" and perform well on high-dimensional datasets, the performance of ABOD implemented on full-dimensional spaces still deteriorates significantly as dimensionality increases, as will be shown in Section 4.1.

The first acknowledged subspace anomaly detection approach for high-dimensional data [32] adopted a grid-based (equi-depth) subspace clustering method, where outliers were searched for in sparse hyper-cuboids rather than dense ones. An evolutionary search (i.e. genetic algorithm) strategy was employed to find sparse grid cells in subspaces. Another feature-bagging technique was used to randomly select subsets from the full-dimensional attributes [33]. Together with some state-of-the-art anomaly detection algorithms, outlier scores in this approach were consolidated in the final step. The major shortcoming of the above two techniques is that the process of selecting subspaces was somewhat arbitrary and a meaningful interpretation as to why a data point is claimed to be an outlier was missing.

To address the above issue, Kriegel et al. proposed the Subspace Outlier Detection (SOD) algorithm, in which for a specific point, variance over different dimensions of the reference set was evaluated [34]. Those dimensions with relatively lower variance were retained to constitute the subspace. Even though it was claimed that the accuracy of SOD in detecting outliers is high, the true positive rate (TPR) is very prone to be reduced if feature scaling is performed beforehand. Another grid-based (equi-width) approach explored subspaces through constructing two bounding constraints, which were defined by information entropy and the density of hypercubes respectively [35]. The algorithm can handle categorical and continuous data simultaneously but suffers from high computational complexity. Moreover, the grid-based segmentation may result in outliers being partitioned into the same hypercube as normal data objects and hence hamper the detection of outliers.

In a $n$-dimensional space, there are $2^{n}$ possible subsets of attributes, i.e. subspaces. The number of possible subspaces grows exponentially with increasing dimensionality. Owing to this combinatorial explosion, exhaustive search over subspaces cannot scale well to high dimensionalities. How to effectively select a meaningful subspace for anomaly detection is still an open research question, which leads to the main motivation of this paper.

\section{Angle-based subspace anomaly detection}

This section firstly elucidates the model assumption, and then introduces the general idea and the process of the ABSAD approach. Afterwards, we elaborate the three main steps of the 
a

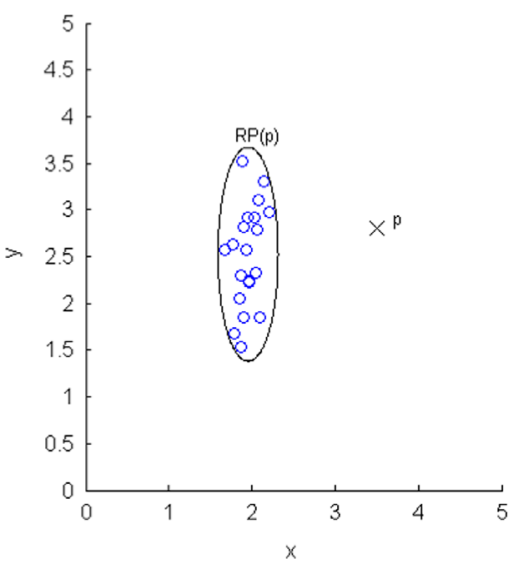

b

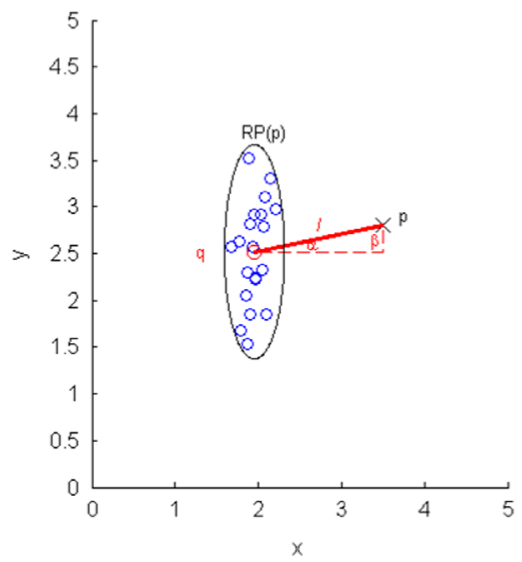

C

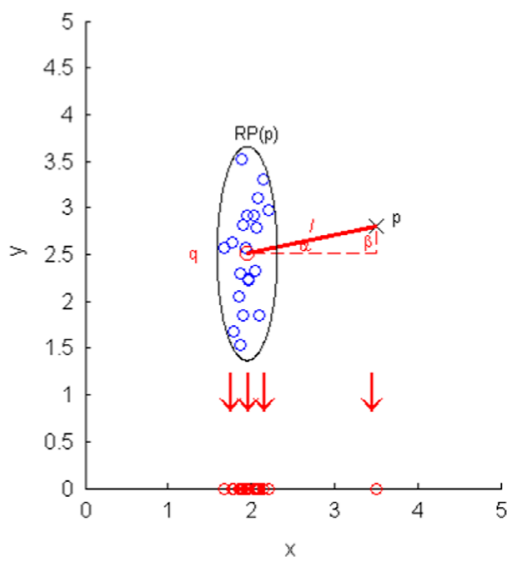

Fig. 3. Intuition of finding relevant subspace and subspace projection.

approach: derivation of reference sets, selection of relevant subspaces and computation to the local outlier score in subspaces. Finally, we consolidate all the steps into a unified algorithm and discuss the choice of input parameters and time complexity of the algorithm.

\subsection{Model assumption}

The separability of different mechanisms may not necessarily depend on the amount of data dimensionality, but instead on the ratio of relevant versus irrelevant attributes [11]. In the cases where the relevant attributes account for a large proportion of the whole dimensions, the separability among different mechanisms tends to increase, which means traditional techniques are still valid and may work even better in high-dimensional spaces. Conversely, when relevant attributes are in a minority of the whole dimensions, the curse of dimensionality would hinder anomaly detection tasks. This paper attempts to address the problem of the latter case. Hereinafter, we assume in this paper the number of anomaly-relevant attributes is in a minority of all the attributes.

\subsection{General idea}

The main purpose of this paper is to compute the degree of deviation of a data point from its neighboring points (i.e. local outlierness) in a meaningful subspace rather than in the full-dimensional space. The subspace is said to be meaningful in the sense that it should be able to capture most of the information with regard to the discordance of an object to its adjacent data instances. By evaluating vectorial angles, we project high-dimensional data onto a lower dimensional axis-parallel subspace that can retain a large portion of a point's local outlier-ness. Subsequently, for each data instance, the degree of deviation from its neighborhood is evaluated on the obtained subspace. And an outlier score is assigned to each of these data points indicating whether the point is an outlier or not. Furthermore, the degree of deviation on each retained dimension for any potential outliers will also serve as a part of the output.

In the following, we define a $m \times n$ matrix $\boldsymbol{X}\left(\boldsymbol{X} \subseteq R^{n}\right)$ as the design matrix. Each row of the matrix represents a data point (also called as data instance, object or observation) in a $n$-dimensional feature space $N$, where $N=\{1, \ldots, n\}$ and $n \geq 2$. The objective of this approach is essentially to define a function that can map $\boldsymbol{X}$ to a real valued vector $L O S$ and a matrix $\boldsymbol{S}$, i.e. $f: \boldsymbol{X} \rightarrow(L O S, \boldsymbol{S})$, where $\operatorname{LOS}_{(i)}$ is the $i$ th point's local outlier score and $\boldsymbol{S}_{(i)}$ contains a set of relevant dimensions of the $i$ th point. To calculate the local outlier-ness of a particular data point $p$, a set of reference points $R P(p)$ of $p$ need to be specified in advance. The set $R P(p)$ reflects the notion of locality.
Additionally, a distance metric $\operatorname{dist}(p, o)$ (e.g. one of the $L_{p}$ norms) measuring the distance between any two points $p$ and $o$ is required when deriving the set $R P(p)$.

Now we will discuss the general idea as to which dimensions should be retained to constitute the subspace to project the original data on. The example shown in Fig. 3 gives us an intuition in selecting relevant subspaces. In a two-dimensional Cartesian coordinate system as shown in Fig. 3(a), the set $R P(p)$ (enclosed by an ellipse) contains the nearest neighbors of an outlier candidate $p$ (black cross). In Fig. 3(b), the geometrical center of $R P(p)$ is calculated and represented by the point $q$ (red circle). Points $p$ and $q$ are connected to form the line $l$ (red solid line). In considering which of the two dimensions ( $x$ and $y$ ) $p$ deviates significantly from its reference points, we can evaluate the angle $\alpha$ between line $l$ and $x$ axis, and $\beta$ between line $l$ and $y$ axis accordingly (both $\alpha$ and $\beta$ are acute angle). Intuitively, the dimension which has a fairly small angle with line $l$ should be retained as the relevant subspace. In this case, angle $\alpha$ is small indicating that line $l$ is nearly parallel to the $x$ axis, whereas $\beta$ is markedly larger in the sense that line $l$ is almost perpendicular to the $y$ axis. Consequently, the dimension on $x$ axis is retained and the dimension on the $y$ axis is excluded in the subspace. Now, as shown in Fig. 3(c), we can project the original data points onto the $x$ axis and compute the local outlier-ness of $p$ in this subspace. The generalization of selecting relevant subspaces in high-dimensional spaces and the concrete method of calculating local outlier score will be described in the subsequent sections.

The process of the proposed approach is as illustrated in Fig. 4. The first step, data preparation, usually encompasses data acquisition, data cleaning, feature selection and other preprocessing procedures. The complexity of this step mainly depends on the quality of the collected raw data. The last step, reporting anomalies, also rests with the requirements of the specific application because there is always a trade-off between false alarm rate and detection rate for unsupervised classification problems. Since these two steps are highly dependent on the concrete applications and plentiful studies have been done specifically on these topics, we will instead mainly focus on the core part of the approach (enclosed by the outer box in Fig. 4) in the remainder of this section.

The feature normalization (or feature scaling) step is to standardize the range of values stored in different features. It is necessary to alleviate the impact exerted by different scales of features. For example, in a real-life problem, different measurements may have different units that could result in diverse scales (such as revolving speed having "rpm" as its unit, whereas displacement is measured in "mm"). Those features with mean or variance that are orders of magnitude larger than others are likely to dominate succeeding computations. Here in the anomaly detection applications, we 


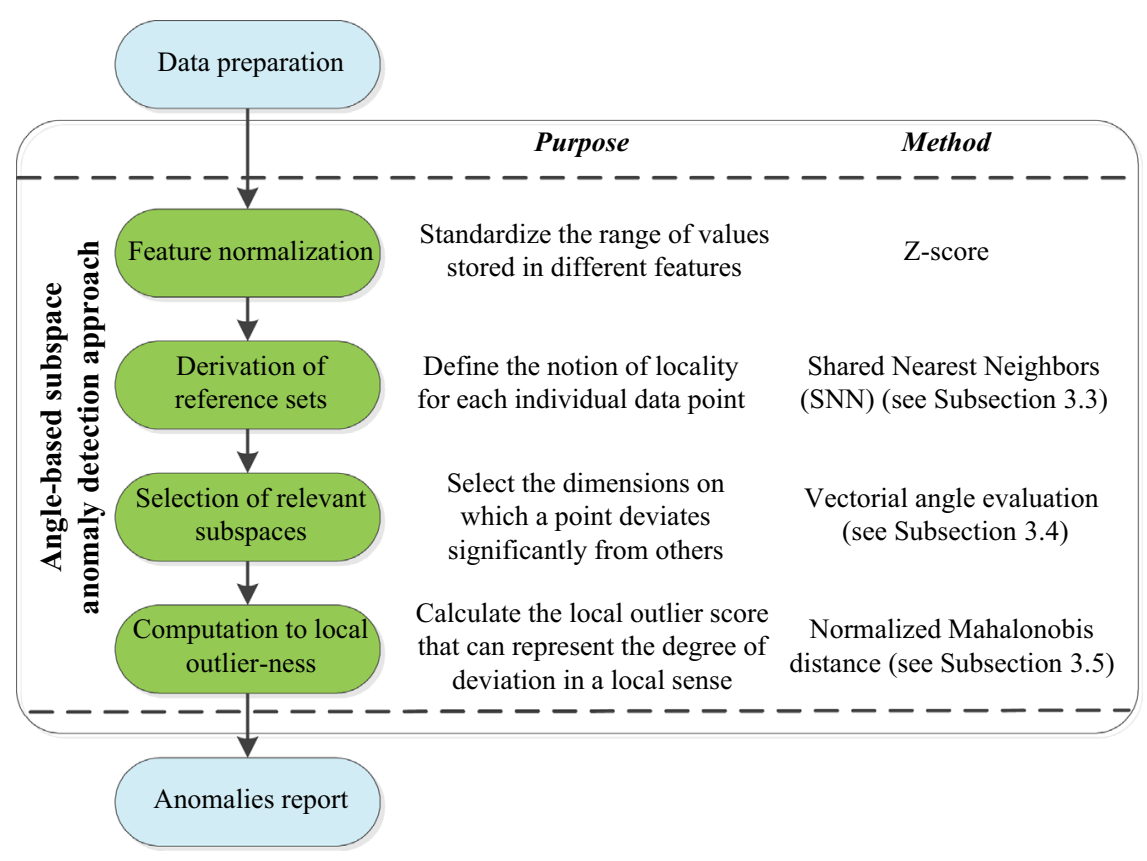

Fig. 4. Process of the angle-based subspace anomaly detection approach.

recommend the use of the Z-score normalization instead of Min-Max scaling for the reason that the latter may suppress the effect of outliers which is inconsistent with our intention. The Z-score method normalizes the design matrix $\boldsymbol{X}$ to a dimensionless matrix $\boldsymbol{X}^{*}$ with zero mean and unit variance. The $i$ th row of $\boldsymbol{X}^{*}$ can be calculated as follows:

$\boldsymbol{X}_{(i)}^{*}=\frac{\boldsymbol{X}_{(i)}-\bar{x}}{\sigma}$, for all $i \in\{1,2, \ldots, m\}$

where $\bar{x}$ is the column-wise mean (a row vector) of the design matrix and $\sigma$ is the column-wise standard deviation (also a row vector) of the design matrix.

The remaining main steps of the approach are basically in line with the example shown in Fig. 3, and we will elaborate these three steps in detail in the next three sections (see Sections 3.33.5).

\subsection{Derivation of reference sets}

Although local outlier detection techniques are more favorable than global ones in terms of accuracy in complex datasets, an additional step needs to be performed to define the implication of locality, i.e. to determine the set of reference points [28]. In lowdimensional spaces, distance-based measures are frequently used to explore the vicinity of a particular point. However, as stated before, in high-dimensional spaces, notions like proximity, distance, or neighborhood become less meaningful as dimensionality increases. To cope with this problem, an alternative series of methods, which introduce a secondary measure based on the rankings of data instances produced by a primary similarity measure, were proposed. Among these methods, the Shared Nearest Neighbors (SNN) approach is the most common one. The applicability of SNN in high-dimensional spaces has been empirically justified in [11] and it was adopted in several other related studies [28,36].

The main idea of the SNN method is that two points generated by the same mechanism should have more overlap in their nearest neighbor list, and vice versa. Specifically, SNN measures the similarity of two points as the number of common nearest neighbors. Prior to calculating the SNN similarity, a primary measure is needed to specify the nearest neighbors for all the points. The primary measure can be any traditional similarity measure (such as $L_{p}$ norm, or the cosine measure). Suppose the $k$ nearest neighbor list of point $p$ is denoted as $N N_{k}(p)$. Notably, the ranking of data instances derived by the primary measure is typically still meaningful in high-dimensional spaces even though the contrast of distance measure has deteriorated with increasing dimensionality. Then, the SNN similarity of point $p$ and point $q$ can be represented as:

$\operatorname{Sim}_{S N N}(p, q)=\operatorname{Card}\left(N N_{k}(p) \cap N N_{k}(q)\right)$

Here the Card function returns the cardinality of the intersection between set $N N_{k}(p)$ and $N N_{k}(q)$. Through sorting all the SNN similarity values of point $p$ and other remaining points in $\boldsymbol{X}, \mathrm{a}$ secondary nearest neighbor list $S N N(p)$ can be derived. The first $s$ elements with largest SNN similarity values in the set $\operatorname{SNN}(p)$, i. e. $S N N_{s}(p)$, constitute the reference set $R P(p)$.

\subsection{Selection of relevant subspaces}

\subsubsection{Definition of the metric "pairwise cosine"}

In the context of detecting anomalies, the selection of a meaningful subspace should retain as much discrepancy between an object and its neighboring points as possible. In Section 3.2, it is stated that when deciding if the $j$ th attribute should be retained as a relevant dimension of the subspace, we can evaluate the acute angle between two lines. The former is the line $l$ connected by an outlier candidate $p$ and the geometrical center $q$ of its reference set $R P(p)$, and the latter is the $j$ th axis. The dimensions that have a comparatively smaller angle with line $l$ constitute the targeted axis-parallel subspace.

To describe the process of selecting relevant subspace formally, let $\vec{\mu}_{n}(j), j \in N$ denote the $j$ th axis-parallel unit vector in a $n$-dimensional space. Furthermore, $\vec{\mu}_{n}(j)$ is a $n \times 1$ column vector with the $j$ th element being one and all the remaining entries being zero. For example, in a five-dimensional space, the axis-parallel unit vector of the $3^{\text {rd }}$ dimension $\vec{\mu}_{5}(3)=\left[\begin{array}{lllll}0 & 0 & 1 & 0 & 0\end{array}\right]^{T}$. Moreover, let $v_{p}$ and $v_{q}$ be the vector representation of point $p$ and $q$ respectively, and $v_{q}$ is the mean vector of all the points in $R P(p)$. Correspondingly, the vector representation of line $l$ can be written 
as $\vec{l}$, and $\vec{l}=v_{p}-v_{q}$. Here we define the $j$ th element of vector $\vec{l}$ as $l_{j}$, i.e. $\vec{l}=\left[l_{1}, l_{2}, \ldots, l_{n}\right]^{T}$.

Since a vector quantity has both magnitude and direction, the angle between two vectors can take values in the range $[-\pi, \pi]$. Instead of calculating the acute angle between two lines, it would be convenient to use a more straightforward metric, the absolute value of the cosine between the two corresponding vectors, to assess the relationship between two lines. We define $\left|\cos \left(\vec{l}, \vec{\mu}_{n}(j)\right)\right|$ as the absolute value of cosine between vector $\vec{l}$ and the $j$ th axis-parallel unit vector $\vec{\mu}_{n}(j)$ :

$\left|\cos \left(\vec{l}, \vec{\mu}_{n}(j)\right)\right|=\frac{\left|<\vec{l}, \vec{\mu}_{n}(j)>\right|}{\|\vec{l}\| \cdot\left\|\vec{\mu}_{n}(j)\right\|}$

In the above formula, $|\cdot|$ is the absolute value sign, $\langle\cdot, \cdot\rangle$ is the scalar product of the interior two vectors and $\|\cdot\|$ calculates the norm of the embedded vector. The absolute value of a cosine function lies in the range $[0,1]$. If the metric is close to one, the $j$ th axis tends to be parallel to line $l$ and should be retained in the subspace. On the contrary, if the metric is approaching zero, the $j$ th axis is prone to be perpendicular to line $l$ and hence should be excluded.

Unfortunately, pairs of random vectors in high-dimensional spaces are typically perpendicular to each other [37]. Specifically, the aforementioned axis-parallel unit vectors and line vector $\vec{l}$ tend to be orthogonal to each other as dimensionality increases. In other words, all cosines of $\vec{l}$ and $\vec{\mu}_{n}(j), j \in N$ will approach zero as $n$ goes to infinity. To verify this, we substitute $\vec{l}=\left[l_{1}, l_{2}, \ldots, l_{n}\right]^{T}$ into formula (3), then we can get,

$\left|\cos \left(\vec{l}, \vec{\mu}_{n}(j)\right)\right|=\frac{\left|l_{j}\right|}{\sqrt{l_{1}^{2}+l_{2}^{2}+\ldots+l_{n}^{2}}}$

If $\left|\cos \left(\vec{l}, \vec{\mu}_{n}(j)\right)\right|, j \in N$ is treated as a random variable, the expectation and variance of it can be calculated as,

$\mathrm{E}\left[\left|\cos \left(\vec{l}, \vec{\mu}_{n}(j)\right)\right|\right]=\frac{1}{n} \cdot \frac{\left|l_{1}\right|+\left|l_{2}\right|+\ldots+\left|l_{n}\right|}{\sqrt{l_{1}^{2}+l_{2}^{2}+\ldots+l_{n}^{2}}}$, where $j \in N$

$\operatorname{Var}\left[\left|\cos \left(\vec{l}, \vec{\mu}_{n}(j)\right)\right|\right]=\frac{1}{n}-\frac{\left(\left|l_{1}\right|+\left|l_{2}\right|+\ldots+\left|l_{n}\right|\right)^{2}}{n^{2} \cdot\left(l_{1}^{2}+l_{2}^{2}+\ldots+l_{n}^{2}\right)}, \quad$ where $j \in N$

Then the following two propositions can be proved (see appendix). The difference of the rate of convergence indicates that the variance converges to zero faster than the expectation. To avoid this statistical fluctuation, an alternative way of assessing the angle between two lines is described subsequently.

Proposition 1. The expectation defined in formula (5) converges to zero as $n$ goes to infinity,

i.e. $\lim _{n \rightarrow \infty}\left(\mathrm{E}\left[\left|\cos \left(\vec{l}, \vec{\mu}_{n}(j)\right)\right|\right]\right)=0$, and the rate of convergence is $1 / \sqrt{n}$.

Proposition 2. The variance defined in formula (6) converges to zero as $n$ goes to infinity,

i.e. $\lim _{n \rightarrow \infty}\left(\operatorname{Var}\left[\left|\cos \left(\vec{l}, \vec{\mu}_{n}(j)\right)\right|\right]\right)=0$, and the rate of con-

vergence is $1 / n$.
Instead of measuring the cosine value of two vectors directly in a $n$-dimensional space, we calculate the average absolute value of cosine between vector $\vec{l}$ and $\vec{\mu}_{n}(j)$ in all possible combinations of two-dimensional spaces. Here the two-dimensional spaces comprise the $j$ th dimension and the $j^{-}$th dimension $\left(j^{-} \in N \backslash\{j\}\right)$, which is selected from all the remaining dimensions in $N$. Obviously, when examining the $j$ th axis with line $l$, there are in total $n-1$ pairs of two-dimensional spaces $\left(j, j^{-}\right)$. Further, we define a new metric PCos (let's call it "pairwise cosine" in the sense that it is derived from two-dimensional spaces) to measure the relationship between a line and an axis in all of the twodimensional spaces. To maintain a uniform notation, let $\operatorname{PCos}\left(\vec{l}, \vec{\mu}_{n}(j)\right)$ denote the "pairwise cosine" between vector $\vec{l}$ and the $j$ th dimension:

$P \operatorname{Cos}\left(\vec{l}, \vec{\mu}_{n}(j)\right)=\frac{1}{(n-1)} \sum_{j^{-}} \in \frac{\left|<\left[\begin{array}{ll}l_{j}^{\#} & l_{j^{-}}^{\#}\end{array}\right]^{T},\left[\begin{array}{ll}1 & 0\end{array}\right]^{T}>\right|}{\left\|\left[l_{j}^{\#} l_{j^{-}}^{\#}\right]^{T}\right\| \cdot\left\|\left[\begin{array}{ll}1 & 0\end{array}\right]^{T}\right\|}$

The above formula is the vector representation of the average absolute value of cosine between line $l$ and the $j$ th axis in all possible combinations of the two-dimensional spaces $\left(j, j^{-}\right)$, where $\left.j^{-} \in N \backslash j\right\}$. In order to avoid a zero denominator, elements in vector $\vec{l}$ that are equal to zero are substituted by a significantly small positive quantity $\varepsilon$ (e.g. $10^{-5}$ ), i.e.

$l_{j}^{\#}=\left\{\begin{array}{cc}l_{j}, & \text { if } l_{j} \neq 0 \\ \varepsilon, & \text { otherwise }\end{array}\right.$ for all $j \in N$

Through simplification, formula (7) can be written as:

$P \operatorname{Cos}\left(\vec{l}, \vec{\mu}_{n}(j)\right)=\frac{1}{(n-1)} \sum_{j^{-} \in N\{\{j\}} \frac{\left|l_{j}^{\#}\right|}{\sqrt{l_{j}^{\# 2}+l_{j^{-}}^{\# 2}}}$

As with the absolute value of cosine in formula (3), the larger the metric PCos is, the more we should include the corresponding dimension in the subspace, and vice versa. Although this very rarely happens in high-dimensional spaces, an exceptional case arises when vector $\vec{l}$ is a zero vector, i.e. vector $v_{p}$ and $v_{q}$ are equivalent or point $p$ and $q$ are overlapping. Intuitively, if point $p$ is exactly the same with the geometric center of its adjacent points, it should not be considered as an outlier. Thus, no subspace should be derived for this point and its outlier score should be zero indicating that it is not abnormal in a local sense.

\subsubsection{Asymptotic property of the metric "pairwise cosine"}

Now we will discuss the expectation and variance of metric "pairwise cosine". Again, if $P \operatorname{Cos}\left(\vec{l}, \vec{\mu}_{n}(j)\right), j \in N$ is regarded as a random variable, its expectation will be as follows:

$$
\mathrm{E}\left[P \operatorname{Cos}\left(\vec{l}, \vec{\mu}_{n}(j)\right)\right]=\frac{1}{n \cdot(n-1)} \sum_{\substack{j, j^{-} \in N \\ j^{-} \neq j}} \frac{\left|l_{j}^{\#}\right|+\left|l_{j^{-}}^{\#}\right|}{\sqrt{l_{j}^{\# 2}+l_{j^{-}}^{\# 2}}}
$$

Notice that PCos is basically the average absolute value of cosine, and it naturally lies in the range $[0,1]$. Therefore, we have the following proposition (proof is provided in the appendix).

Proposition 3. The expectation in formula (9) lies in the interval $(1 / 2, \sqrt{2} / 2]$ and does not depend on the magnitude of dimensionality.

Besides, the expectation and variance of PCos tend to be asymptotically stable along with the increase of dimensionality. 
a

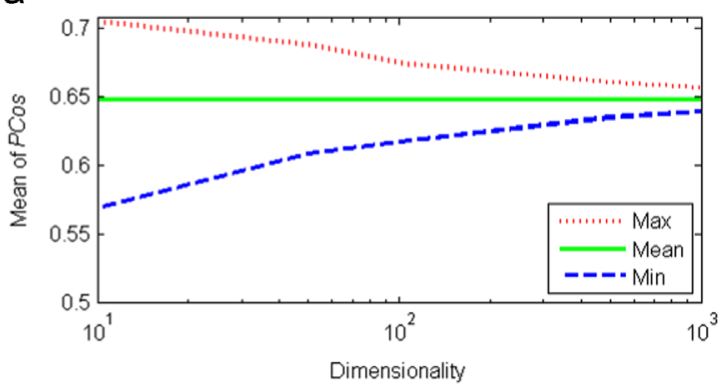

C

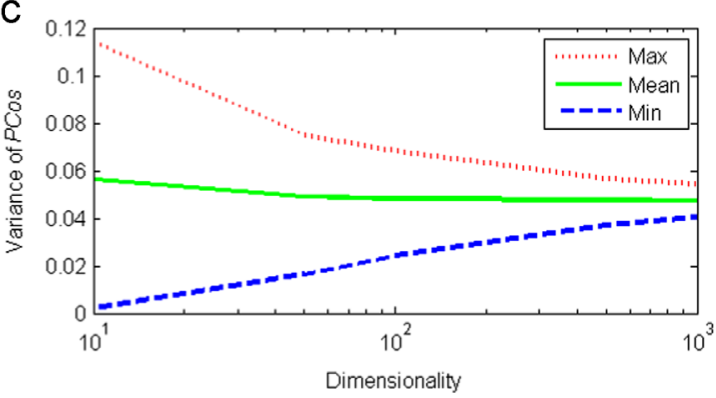

b

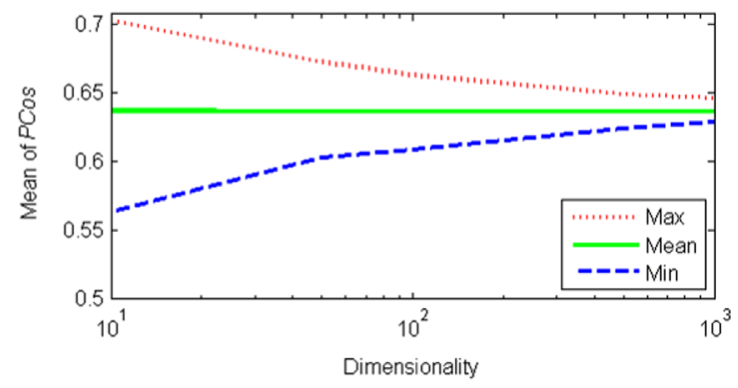

d

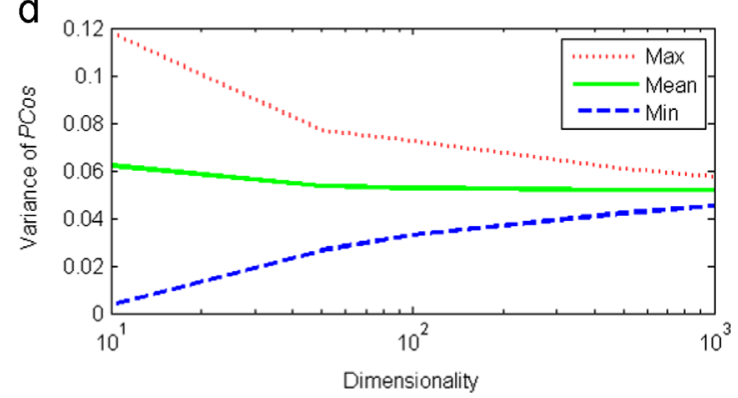

Fig. 5. Asymptotic property of expectation and variance of PCos.

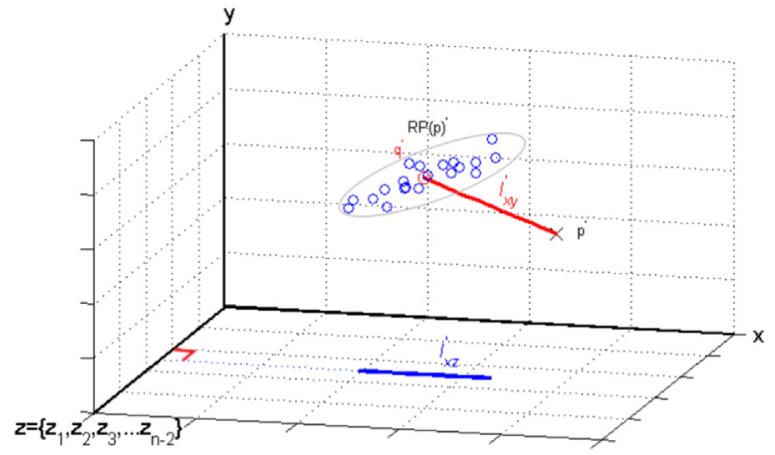

Fig. 6. An illustration to the capability of the metric "pairwise cosine" to capture correlated relevant attributes.

As shown by (a) and (b) in Fig. 5, the mean of PCos that is derived from a uniformly distributed dataset and a normally distributed dataset, both with $10^{5}$ samples, are plotted against increasing dimensionalities and gradually converge to a value around 0.65 (not exactly). Even though the variance of this metric is analytically intractable from our knowledge, as demonstrated by (c) and (d) in Fig. 5, it again tends to level off and be rather stable as dimensionality increases. Notably, the asymptotic property of the expectation and variance of the metric PCos holds even for samples with a large order of magnitude based on our experiments.

\subsubsection{Ability of the metric to capture correlated relevant attributes}

It is common to see the presence of correlations between attributes in real-world applications. Traditional anomaly detection techniques usually use decorrelation methods such as PCA to obtain a new set of orthogonal basis prior to detecting anomalies. Under the assumption of this paper, the metric PCos can capture relevant attributes even if these relevant attributes are correlated to each other.

To explain the above statement, we simply extend the twodimensional example described in Section 3.2 to a multidimensional case as shown in Fig. 6, in which $x$ and $y$ are the two dimensions relevant to the abnormality of point $p$ and other dimensions $z=\left\{z_{1} z_{2}, z_{3}, \ldots, z_{n-2}\right\}$ are irrelevant. Unlike the previous example, correlation between relevant attributes is introduced in this case, which can be seen from the projection of original objects on the plane spanned by the $x$ and $y$ dimension. The projection of line $l$ on the two-dimensional space $(x, y)$ is $l_{x y}^{\prime}$ (the red line), and the nominal projections of line $l$ on other twodimensional spaces $\left(x, z_{1}\right),\left(x, z_{2}\right),\left(x, z_{3}\right), \ldots,\left(x, z_{n-2}\right)$ are represented by $l_{x z}^{\prime}$ (the blue line). Since dimensions $z=\left\{z_{1} z_{2}, z_{3}, \ldots, z_{n-2}\right\}$ are irrelevant to the abnormality of point $p$ from its surrounding points, we can postulate that most of the lines $l_{x z}^{\prime}$ tend to be approximately orthogonal to axis $z$, thereby being parallel to axis $x$. To calculate the $P C o s$ value between line $l$ and the $x$ axis, we need to average all the absolute values of cosines between line $l_{x y}^{\prime}, l_{x z_{1}}^{\prime}, l_{x z_{2}}^{\prime}, \ldots, l_{x z_{n-2}}^{\prime}$ and axis $x$ in the corresponding two-dimensional spaces. The existence of correlation between relevant attributes may lead to a small cosine value between line $l_{x y}^{\prime}$ and the $x$ axis in the two-dimensional space $(x, y)$. But the cosine values between $l_{x z_{1}}^{\prime}, l_{x z_{2}}^{\prime}, \ldots, l_{x z_{n-2}}^{\prime}$ and axis $x$ in other two-dimensional spaces should be large, and hence raise the value of PCos value between line $l$ and the $x$ axis. Based upon the assumption that relevant attributes are in a minority of all the dimensions, we can expect to accurately locate anomaly-relevant attributes by using the proposed metric even though there are correlations between these attributes.

\subsubsection{Filtering step for selecting relevant dimensions}

As elaborated before, the dimensions with large PCos values should be incorporated into the subspace, whereas the dimensions with small $P C O S$ values should be excluded. For each data point in the dataset $\boldsymbol{X}$, there is one particular line $l$ and we can obtain $n$ different values of $P C O$ s between vector $\vec{l}$ and diverse dimensions by applying formula (8) iteratively. It has been justified that PCos is a relatively robust metric in high-dimensional spaces, so for a specific data point we can single out those large PCos values by setting a threshold $G$ as below:

$G=(1+\theta) \cdot \frac{1}{n} \sum_{j \in N} P \operatorname{Cos}\left(\vec{l}, \vec{\mu}_{n}(j)\right)$ 
Here, $\theta$ is an input parameter lying in $[0,1)$, and the right part of the multiplier in formula (10) is the average PCos over all the dimensions. Those dimensions with a PCos value greater than $G$ are retained to constitute the relevant subspace for a specific data point. Further, we can save the obtained subspace index into $\boldsymbol{S}$, which is a $m \times n$ matrix. Concretely, the $i$ th row of the matrix $\boldsymbol{S}_{(i)}$ stores the relevant subspace of the corresponding $i$ th data point, and the $j$ th column defines whether the $j$ th attribute should be retained. We suppose $\boldsymbol{S}_{(i)}(j)$ locates to the cell of the $i$ th row and the $j$ th column. If we are considering the $i$ th data point, and the $j$ th attribute is singled out to be retained, then $\boldsymbol{S}_{(i)}(j)=1$, otherwise $\boldsymbol{S}_{(i)}(j)=0$.

\subsection{Computation to local outlier-ness in subspaces}

After going through the process of selecting relevant subspace for each data point, we might find that some of the points do not have any relevant attributes retained as a part of the subspace. In other words, when the $i$ th point conforms to this situation, $\boldsymbol{S}_{(i)}(j)$ equals to zero for all $j \in N$. This circumstance shows that the $i$ th point does not significantly deviate from its neighbors in any subsets of all the dimensions. To explain this, we can imagine that if a point does deviate from its neighbors in a subset of all the attributes, there should be some extreme values in the "pairwise cosine" that would be captured by the filtering step. Thus, for those points with no subspace to project on, we simply set the outlier score to zero.

In the following, we describe how to measure the local outlierless of a data point in its subspace. Normally, some state-of-the-art techniques (e.g. distance-based, density-based, statistical models) which can effectively and efficiently detect anomalies in lowdimensional spaces are employed to calculate the overall outlierness at this stage. For example, in the SOD algorithm [34], Euclidean distance is utilized to calculate the local outlier-ness of a point in the subspace projection. Generally, these methods are also applicable in our algorithm and can perform well if the outlier score is normalized in a good manner. However, a single outlier score can neither reveal the degree of deviation in each individual dimension for an outlier nor provide a rank to the degree of deviation in these dimensions. Hereinafter, we compute the local outlier-ness of an outlier candidate in two steps: measure the overall local outlier score; calculate its degree of deviation on each individual dimension of the obtained subspace. By doing so, not only a comprehensive outlier score can be derived but also a preliminary interpretation as to why a point is classified as an outlier can be provided.

\subsubsection{Overall local outlier score}

Due to the possible existence of correlation between the retained dimensions, we introduce a normalized Mahalanobis distance to measure the overall local outlier score in the subspace for a specific data point. Firstly, let $\boldsymbol{X}_{(i)}^{* \prime}$ be the vector representation (a row vector) of the $i$ th normalized point's projection on the retained subspace, and $R P(i)^{\prime}$ denotes the set of the projected reference points of the original reference points $R P(i)$ on the retained subspace. Secondly, the mean vector of the reference point's projection is denoted as $\overline{R P(i)^{\prime}}$ (also a row vector), and the inverse of the covariance matrix of the projected reference points $R P(i)^{\prime}$ is $\boldsymbol{\Sigma}_{R P(i)^{\prime}}^{-1}$. Further, let $d(i)$ denote the number of retained dimensions for the $i$ th data point. Then the local outlier score for the $i$ th point $\operatorname{LOS}(i)$ is defined as follows:

$\operatorname{LOS}(i)=\frac{1}{d(i)} \cdot \sqrt{\left(\boldsymbol{X}_{(i)}^{* \prime}-\overline{R P(i)^{\prime}}\right) \Sigma_{R P(i)^{\prime}}^{-1}\left(\boldsymbol{X}_{(i)}^{* \prime}-\overline{R P(i)^{\prime}}\right)^{T}}$

In formula (11), the right side of the multiplier is essentially the Mahalanobis distance from the normalized point $i$ to its reference points in the subspace projection. Essentially, the local outlier score for the $i$ th point $\operatorname{LOS}(i)$ is the Mahalanobis distance in the retained subspace normalized by the number of retained dimensions.
Notably, for the covariance matrix of the projected reference points $\boldsymbol{\Sigma}_{R P(i)^{\prime}}$ to be invertible, the covariance matrix should be nonsingular. The non-singularity of the covariance matrix relies on the following three prerequisites: (i) in the data preparation step, feature selection has eliminated redundant attributes and the retained dimensions in the subspace are not highly correlated; (ii) we assumed that the anomaly-relevant attributes are in a minority of all the dimensions in Section 3.1 and the process of selecting relevant subspaces described in Section 3.4.4 should filter out large amount of anomaly-irrelevant attributes, and hence the number of retained dimensions is small; (iii) the choice of the number of reference points $s$ (as will be discussed in Section 3.6.2) should be set large enough. The above three conditions can basically suffice for the nonsingularity of the covariance matrix

\subsubsection{Degree of deviation on each individual dimension}

Now we will discuss the calculation to the degree of deviation on each individual dimension of the obtained subspace. Intuitively, the overall local outlier score $\operatorname{LOS}(i)$ can be viewed as the length of the line connected by the $i$ th normalized point and its reference points in the subspace projection, i.e. the length of the line $\boldsymbol{X}_{(i)}^{* \prime}-\overline{R P(i)^{\prime}}$. For the $i$ th point, the degree of deviation on the retained $j$ th dimension $\operatorname{LOS}(i, j)$ can be measured by the length of projection from the above line onto the $j$ th dimension in the subspace. To adhere to a consistent notation, let $\vec{\mu}_{d(i)}(j)$ denote the $j$ th axis-parallel unit vector in the $i$ th point's retained subspace and $\gamma_{i j}$ be the angle between the line $\boldsymbol{X}_{(i)}^{* \prime}-\overline{R P(i)^{\prime}}$ and the $j$ th axis of the subspace. Then $\operatorname{LOS}(i, j)$ can be calculated as below:

$\operatorname{LOS}(i, j)=\left|\cos \left(\gamma_{i j}\right)\right| \cdot \operatorname{LOS}(i)=\frac{\left|\left\langle\boldsymbol{X}_{(i)}^{* \prime}-\overline{R P(i)^{\prime}}, \vec{\mu}_{d(i)}(j)\right\rangle\right|}{\left\|\boldsymbol{X}_{(i)}^{* \prime}-\overline{R P(i)^{\prime} \|} \cdot\right\| \vec{\mu}_{d(i)}(j) \|} \cdot \operatorname{LOS}(i)$

The expanded form of $\left|\cos \left(\gamma_{i j}\right)\right|$ in formula (12) is similar to formula (3) and the metric $\operatorname{LOS}(i, j)$ is essentially to measure the length of the projection from one vector to another vector in the subspace. Due to the triangle inequality, the obtained local outlier score on each individual dimension is not comparable with the overall local outlier score. Furthermore, the single-dimensional local outlier score cannot be compared across different points. But for a particular data point, the difference of these single-dimensional local outlier scores of one point can reflect the magnitude of influence to the overall local outlier-ness contributed by different dimensions.

At this stage, we are able to discern anomaly-relevant attributes from matrix $\boldsymbol{S}$ for a particular point. In the case of several dimensions being retained, a rank to these dimensions according to the single-dimensional local outlier score might facilitate further investigation as to the cause of abnormality. Therefore, an update policy applied to matrix $\boldsymbol{S}$ can be implemented after calculating all the local outlier scores. The update policy is described as follows:

$\boldsymbol{S}_{(i)}(j)=\left\{\begin{array}{cc}\operatorname{LOS}(i, j), & \text { if } \boldsymbol{S}_{(i)}(j)=1 \\ 0, & \text { otherwise }\end{array}\right.$

Now from matrix $\boldsymbol{S}$, through ranking those non-zero elements in a row, one can provide a preliminary explanation as to why a data point is declared to be an anomaly.

\subsection{Model integration and discussion}

In the preceding sections, we described the main steps of the ABSAD algorithm, especially the procedure for finding relevant subspace by analyzing vectorial angles in high-dimensional spaces. In the following, we streamline the above steps and consolidate 


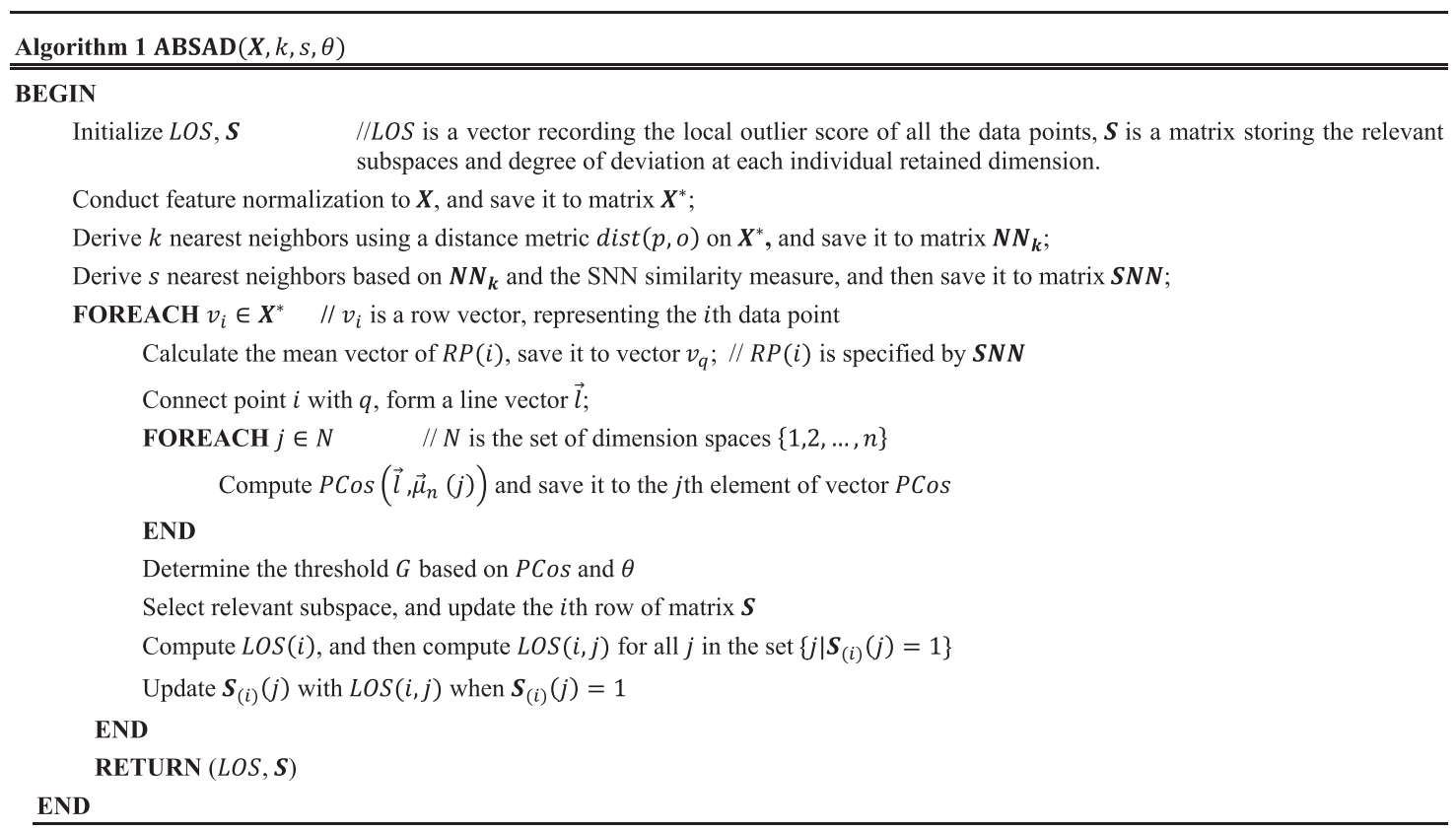

Fig. 7. The angle-based subspace anomaly detection algorithm

them into a unified algorithm, and then discuss the choice of input parameters and time complexity of the algorithm.

\subsubsection{Model integration}

A panoramic view of the algorithm is presented in Fig. 7. All of the other procedures in Fig. 7 have been covered in previous sections except for the way of determining whether a given data point is an anomaly or not based upon its overall local outlier score. In general, anomaly detection can be considered as a skewed binary classification problem. Unfortunately, there is no deterministically accurate criterion to convert the continuous local outlier score to a binary label, namely normal or abnormal. The simplest way is to treat the top-most data points with the largest local outlier scores as anomalies and the number of anomalous points is given by the end-user. Another way is to set a threshold, and the data point which has a local outlier score exceeding the threshold should be regarded as an anomaly. If we have labeled data, especially some anomalous examples, the threshold can also be set through model selection as is frequently done in supervised learning problems. Of course, a classifier with reject option can also be employed here to refuse to classify any given objects as abnormal or normal. After all, the objective of introducing one additional threshold is to achieve higher precision and recall, i.e. to reduce the probability of committing both type I (false positive) and type II (false negative) error.

\subsubsection{Input parameters}

As shown in Fig. 7, in addition to the design matrix, the algorithm takes in three manually specified parameters. Firstly, parameter $k$ specifies the number of nearest neighbors for computing SNN similarity. As with some other algorithms related to the SNN method, $k$ should be set large enough so as to capture sufficient points from the same generating mechanism. As reported in [11], if $k$ is chosen roughly in the range of cluster size then a considerably satisfactory performance in terms of defining the notion of locality can be achieved. Secondly, parameter $s$ defines the size of the reference sets. For the same reason, it should be chosen large enough but not greater than $k$. In [11], it was justified that the performance of the SNN method does not degrade until the size of reference points approaches the full dataset size. Last but not least, $\theta$ decides which dimensions should be kept as a part of the relevant subspace. It may have a great influence on selecting relevant subspace and hence affect the subsequent calculation of local outlier score. Generally, the lower value $\theta$ is, the more dimensions will be included in the subspace, and vice versa. Since the aforementioned "pairwise cosine" is a relatively steady metric with regard to the number of dimensionalities, $\theta$ can be tuned in some relatively low-dimensional space and then put into use for concrete applications with higher dimensionalities. In our experiment, $\theta$ was set to 0.45 and 0.6 for the synthetic datasets and real-world dataset respectively, and consistently good results were produced. So we would recommend setting the parameter $\theta$ accordingly.

In addition, as mentioned in Section 3.5, the choice of $s$ and $\theta$ will directly affect subspace projection of the $i$ th points' reference points $R P(i)^{\prime}$, thereby deciding whether the covariance matrix $\Sigma_{R P(i)}$ ' is invertible or not. Concretely, the number of rows in the reference points $R P(i)^{\prime}$ is equal to $s$ and the number of columns is negatively correlated to the parameter $\theta$. Under the assumption that anomaly-relevant attributes take only a small portion of all the dimensions, the number of retained dimensions in the subspace will not be too large if an appropriate $\theta$ is chosen. Together with a large enough $s$, we can expect the number of rows of the reference points $R P(i)^{\prime}$ to be greater than the number of columns, and hence one of the necessary conditions for the covariance matrix $\Sigma_{R P(i)}$, to be invertible is satisfied.

\subsubsection{Time complexity}

Now we will discuss the time complexity of the proposed algorithm. Firstly, feature normalization using the Z-score method takes $\mathrm{O}(m)$ assuming $m$ is considerably larger than $n$. Then, deriving $k$ nearest neighbors and $s$ nearest neighbors have the time complexity of $\mathrm{O}\left(m^{2} \cdot \max (n, k)\right)$ and $\mathrm{O}(m \cdot k)$ respectively. Finally, the step of finding relevant subspace and calculating local outlier score takes $\mathrm{O}\left(m \cdot n^{2}\right)$. In summary, the time complexity of the algorithm is in $\mathrm{O}\left(m^{2} \cdot \max (n, k)\right)$ considering $m$ is typically much larger than $n$ and $k$. If some indexing structures like k-d tree or $\mathrm{R}^{*}$ tree are employed here, the complexity can be reduced 
to $\mathrm{O}(m \log m \cdot \max (n, k))$, which is rather attractive compared with most of the existing high-dimensional anomaly detection techniques.

\section{Numerical illustration}

In this section, we evaluate the proposed algorithm on both synthetic data and a real-world dataset, and further contrast the proposed algorithm with several prominent outlier detection techniques. There is a wide range of outlier detection techniques including all the variants that can be chosen for comparison. We select LOF, ABOD and SOD as the competitors for the reason that: (i) the LOF approach is one of the most well-known local outlier detection techniques that measures the density of a point in a local sense; (ii) the ABOD approach is an angle-based, global outlier detection approach which was claimed to be still effective in highdimensional spaces; (iii) the SOD approach is the most similar algorithm to our suggested algorithm but it selects subspaces based on the variance of the reference points on different dimensions. Through utilizing the well-established Receiver Operating Characteristic (ROC) curve, accuracy indicators comprising of True Positive Rate (TPR) and False Positive Rate (FPR) for each of the approaches are compared in different dimensionality settings.

To study the performance of the suggested algorithm in a varying dimensionality environment, the dimensions of the experimental dataset need to be adjustable. Besides, attributes that are irrelevant to any data-generating mechanisms should be known in advance in order to verify the veracity of the retained subspace, whereas this is typically not explicit for most of the realworld datasets. Therefore, we construct a series of synthetic datasets with changing dimensionalities to validate the effectiveness of the suggested algorithm and thus compare it with other techniques. Afterwards, we verify the applicability of the algorithm on a real-world dataset.

\subsection{Validation using synthetic datasets}

\subsubsection{Data generation}

In previous sections, we mentioned that the suggested algorithm can detect anomalies in meaningful subspaces, i.e. subsets of attributes related to different data-generating mechanisms. To clarify this point, we designed two different data-generating mechanisms which can influence two non-overlapping subsets of all the attributes separately. Then we placed several outliers that deviate from ordinary data generated by these mechanisms in the final dataset, similar to the case used in [34].

Specifically, for simplicity, a two-dimensional Gaussian distribution with $\mu_{1}=0.5$ and $\sigma_{1}=0.12$ at each dimension serves as the first generating mechanism, and a three-dimensional Gaussian distribution with $\mu_{2}=0.5$ and $\sigma_{2}=0.04$ at each dimension is employed as the second generating mechanism. The remaining irrelevant attributes contain values that are uniformly distributed in the range $[0,1]$. To make this example more generic, we deliberately set the variance of the two Gaussian distributions differently than the variance of the irrelevant attributes, which follow the standard uniform distribution $\left(\sigma_{u}^{2}=1 / 12 \approx 0.0833\right)$. Through varying the number of irrelevant attributes, a series of datasets with dimensionalities of different sizes can be constructed. For example, 95 irrelevant attributes together with the data generated by the two Gaussian distributions give rise to a 100-dimensional dataset. In our experiment, different dimensional settings including 40, 70, 100, 400, 700, 1000 dimensions were tested.

Further, for each of the two Gaussian distributions, 480 rows of normal data and 20 rows of abnormal data are generated. The maximum distances from the normal data to the cluster centers of the two Gaussian distributions are 1.23 and 0.87 respectively. The distance from the anomalies to the centers of the two Gaussian distributions lies in the range $[1.5,1.7]$ and $[1.1,1.3]$ accordingly. Through rearranging the location of the normal and abnormal data and concatenating all the above data with the uniformly distributed data values, the final dataset with 470 rows of normal data and 30 rows of abnormal data is obtained. The layout of the constructed dataset is shown in Fig. 8, in which the last 30 rows of the dataset can be considered as anomalies in different subspaces. Notice that the last 10 rows of the dataset deviate from normal data in the features where both the two-dimensional Gaussiandistributed data and the three-dimensional Gaussian-distributed data were generated. An ideal subspace anomaly detection technique should be able to detect these anomalies and also report the corresponding subspaces correctly. Additionally, the parameter $k, s$ and $\theta$ were set to be 110, 100 and 0.45 respectively.

\subsubsection{Simulation results and discussions}

The experimental results indicate that the suggested algorithm outperforms the other three in various high-dimensional settings. The comparison of the accuracy of the four algorithms in three different dimensionality settings is presented in Fig. 9. Even though LOF and $A B O D$ are very effective in low-dimensional spaces, their accuracy deteriorates considerably as dimensionality increases. The SOD approach does not behave as it was reported because the variance of the two-dimensional Gaussian distribution exceeds the variance of the remaining uniformly distributed attributes significantly which leads to the algorithm avoiding the selection of the first two dimensions as the aiming subspaces. As expected, the accuracy of the proposed algorithm is rather stable as the number of dimensions increases. Notably, even in 1000-dimensional spaces, our algorithm can still provide a satisfactory accuracy with the value of Area under Curve (AUC) up to 0.9974 (the closer to 1, the better of the accuracy)

Apart from the superior accuracy, the algorithm can also accurately recognize the dimensions on which anomalies deviate substantially from their adjacent points. The last 30 rows of matrix $\boldsymbol{S}$ corresponding to the 30 anomalies are listed in Fig. 10, in which the dimensions related to different generating mechanisms are separated by vertical lines, and different sets of rows ( 471 to 480,481 to 490 , and 491 to 500) are separated by horizontal lines. A zero entry in the matrix implies that the corresponding dimension has a relatively small value of $P C O S$ and was not retained in the subspace for the specific data point. A non-zero entry not only signifies the dimension is a part of the subspace but also reflects the degree of deviation on this single dimension for the particular observation. As indicated by Fig. 10, the retained subspaces match precisely with the dimensions where the abnormal data were placed. Moreover, the rank of the non-zero elements in a row can give us a primary understanding on the magnitude of contribution to abnormality made by different retained dimensions.

The above experiment demonstrated the suggested way of selecting relevant subspaces can largely retain the discrepancy between points and their neighboring points. Generally, the metric PCos has large values in relevant dimensions, and small values in irrelevant dimensions. It is precisely the difference between PCos values in relevant and irrelevant dimensions which gives us the possibility to find a meaningful subspace. To our surprise, the increase of irrelevant attributes in this experiment does not impede the selection of relevant subspaces; instead, it serves as a foil and helps to accurately locate relevant attributes. Specifically, when the ratio of relevant versus irrelevant attributes is small, we can expect distinguishing values between different PCos of relevant and irrelevant attributes even though the total dimensions are enormous. This phenomenon seems to reflect the "blessing of dimensionality" accompanied by the "curse of dimensionality" [5]. Notably, if a striking contrast between different PCos values exists, it is easy to select the relevant subspace. However, 


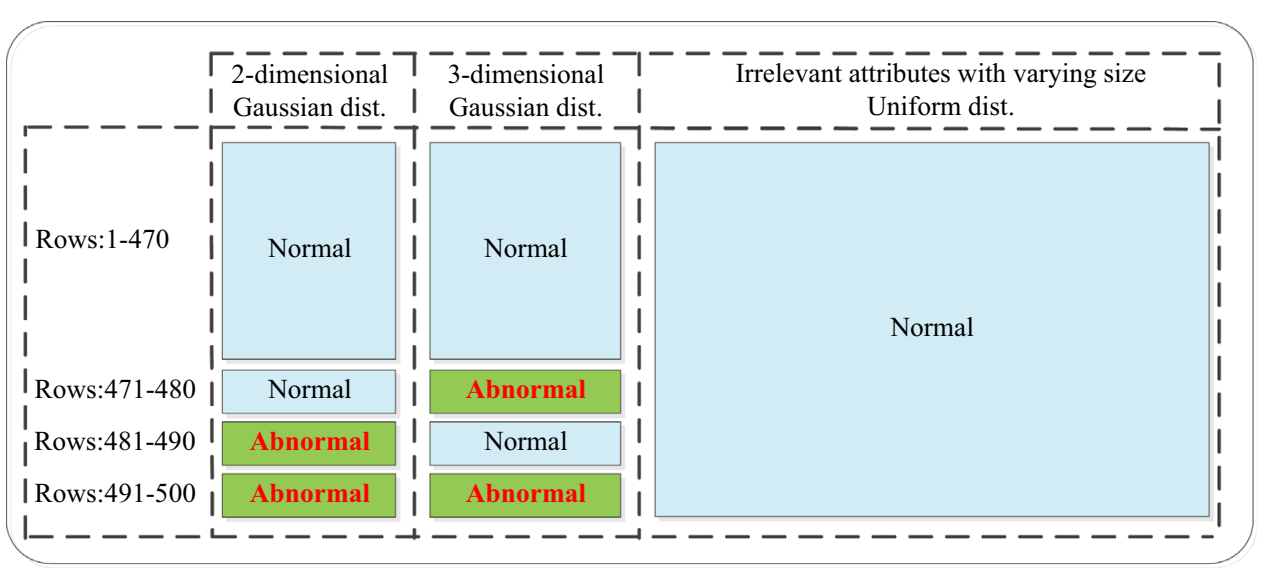

Fig. 8. Layout of the synthetic datasets.

a

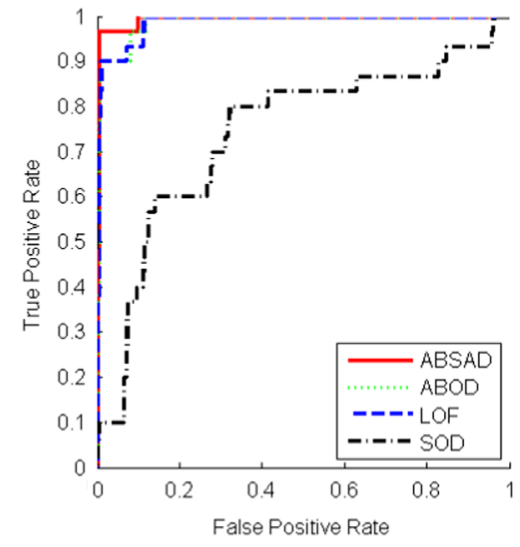

b

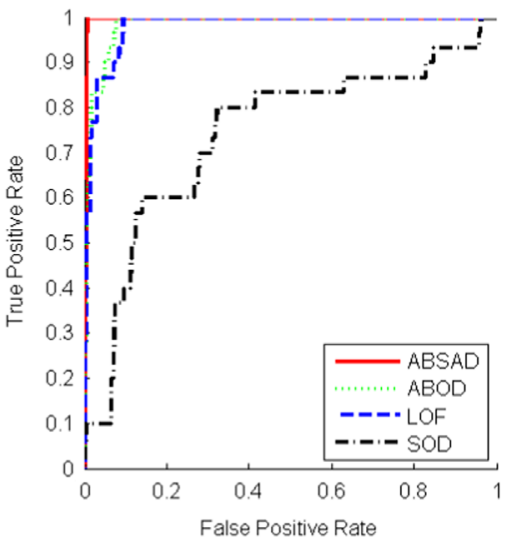

C

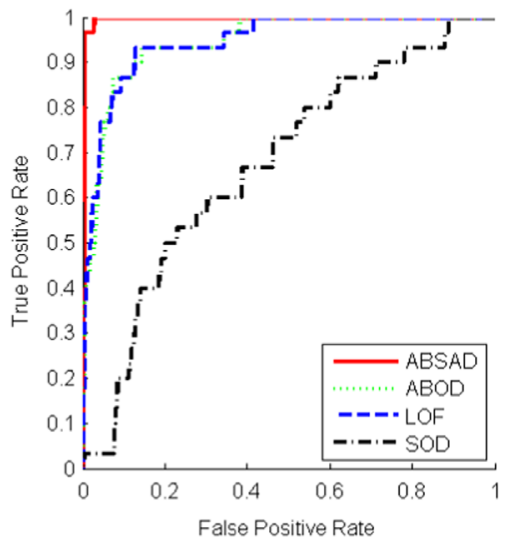

Fig. 9. ROC curve comparison on different dimensionality settings.

if there are insufficient differences between $P C$ os values, the approach might not end up with ideal subspaces. Under this circumstance, many traditional anomaly detection approaches may work even better than this type of subspace anomaly detection technique, as identified by previous studies [11].

\subsection{Verification by a real-world dataset}

In a Swedish hydropower plant, 128 analog (which will be used in this example) and 64 digital transducers have been deployed on one of their hydro-generator units. Different signals are captured periodically from scattered parts of the unit, such as rotor rotational velocity, shaft guide bearing temperature, hydraulic oil level and so on. The existing way of monitoring the health status of the unit on the system level is to manually set thresholds on several critical features. Once the gathered values exceed these thresholds, an alarm is triggered and a work order is then created in the Computerized Maintenance Management System (CMMS). This rigid method of fault detection is highly dependent on threshold settings and can only detect some obvious abnormalities.

In this application, different faults of the unit, caused by diverse factors, are normally embodied in small subsets of all the features. In Table 1 , some of the known faults of the unit are listed, and relevant measurements corresponding to each fault are also presented. Considering the total number of measurement points, each of these sets of relevant measurements accounts for only a small proportion of the whole features, which is consistent with our assumption described in Section 3.1. In addition, for different faults, their relevant subsets of features can overlap with each other or be utterly nonintersected. In other words, each measurement can be indicative of one or more than one fault.

Under different ambient environment and operational loads, multi-dimensional measurements that possess different statistical characteristics may vary greatly. Without losing generality (the algorithm can be adapted to an online mode to capture timevarying characteristics of the system), we considered the problem of detecting faults in the case when the generator unit is running in a steady-state, which is also known as conditional or contextual anomaly detection [25]. To conduct conditional anomaly detection, environmental attributes and indicator attributes first need to be differentiated. As defined in [38], environmental attributes do not directly indicate anomalies but define the context of different datagenerating mechanisms. In contrast, indicator attributes can be indicative of anomalous observations. In this example, we treat the attribute "opening percentage of the inlet vanes" as the environmental attribute for the reason that it modulates the flow of water to meet system capacity requirements and hence impacts other attributes primarily. Through feature selection, 102 out of the original 128 measurements were chosen to be the indicator attributes. For simplicity, we discretize the environmental attributes and define the context to be a specific range of opening percentage of the vanes' position. For example, one context is defined as "the vanes' opening percentage is in the range from $72 \%$ to $73 \%$ ". Finally, a dataset with 1000 ordinary samples and 10 abnormal data, placed in the rows from 1001 to 1010, was constructed. These data were generated in the same context and abnormal data were selected 


\begin{tabular}{|c|c|c|c|c|c|c|c|c|}
\hline & 0 & 0 & 0 & 0 & 9.9 & $\ldots$ & 0 & $\ldots$ \\
\hline & 0 & 0 & 0 & 0 & 21.73 & $\ldots$ & 0 & $\ldots$ \\
\hline & 0 & 0 & 8.86 & 0 & 6.18 & $\ldots$ & 0 & $\ldots$ \\
\hline & 0 & 0 & 3.91 & 0 & 6.98 & $\ldots$ & 0 & $\ldots$ \\
\hline & 0 & 0 & 0 & 19.4 & 0 & $\ldots$ & 0 & $\ldots$ \\
\hline & 0 & 0 & 21.35 & 0 & 0 & $\ldots$ & 0 & $\ldots$ \\
\hline & 0 & 0 & 0 & 0 & 9.62 & $\ldots$ & 0 & $\ldots$ \\
\hline & 0 & 0 & 0 & 8.55 & 6.12 & $\ldots$ & 0 & $\ldots$ \\
\hline & 0 & 0 & 0 & 23.93 & 0 & $\ldots$ & 0 & $\ldots$ \\
\hline & 0 & 0 & 14.6 & 0 & 0 & $\ldots$ & 0 & $\ldots$ \\
\hline & 0 & $\overline{13.41}$ & 0 & 0 & 0 & $\ldots$ & 0 & $\ldots$ \\
\hline & 0 & 13.59 & 0 & 0 & 0 & $\ldots$ & 0 & $\ldots$ \\
\hline & 19.04 & 0 & 0 & 0 & 0 & $\ldots$ & 0 & $\ldots$ \\
\hline & 0 & 0 & 0 & 0 & 0 & $\ldots$ & 0 & $\ldots$ \\
\hline $\mathbf{S}(71.500 \cdot)=$ & 17.82 & 0 & 0 & 0 & 0 & $\ldots$ & 0 & $\ldots$ \\
\hline $\mathbf{S}(4 / 1: 500,:)=$ & 12.49 & 0 & 0 & 0 & 0 & $\ldots$ & 0 & $\ldots$ \\
\hline & 7.28 & 5.17 & 0 & 0 & 0 & $\ldots$ & 0 & $\ldots$ \\
\hline & 10.39 & 0 & 0 & 7.65 & 0 & $\ldots$ & 0 & $\ldots$ \\
\hline & 0 & 15.44 & 0 & 0 & 0 & $\ldots$ & 0 & $\ldots$ \\
\hline & 14.77 & 0 & 0 & 0 & 0 & $\ldots$ & 0 & $\ldots$ \\
\hline & 0 & 7.61 & 7.72 & 0 & 8.09 & $\ldots$ & 0 & $\ldots$ \\
\hline & 0 & 6.3 & 0 & 9.58 & 0 & $\ldots$ & 0 & $\ldots$ \\
\hline & 9.95 & 0 & 0 & 12.57 & 0 & $\ldots$ & 0 & $\ldots$ \\
\hline & 0 & 9.65 & 8.85 & 0 & 7.81 & $\ldots$ & 0 & $\ldots$ \\
\hline & 9.05 & 0 & 0 & 6.05 & 8.76 & $\ldots$ & 0 & $\ldots$ \\
\hline & 0 & 12.54 & 0 & 10.48 & 11.06 & $\ldots$ & 0 & $\ldots$ \\
\hline & 8.36 & 0 & 5.93 & 5.74 & 6.05 & $\ldots$ & 0 & $\ldots$ \\
\hline & 0 & 11.06 & 8.34 & 10.79 & 0 & $\ldots$ & 0 & $\ldots$ \\
\hline & 0 & 5.52 & 4.34 & 0 & 6.09 & $\ldots$ & 0 & $\ldots$ \\
\hline & 0 & 9.26 & 8 & 9.78 & 0 & $\ldots$ & 0 & .. \\
\hline
\end{tabular}

Fig. 10. Local outlier score on each individual retained dimension.

Table 1

Relevant attributes corresponding to some of the faults of the generator unit

\begin{tabular}{|c|c|c|c|c|}
\hline \multirow[b]{2}{*}{ Functional location } & \multirow[b]{2}{*}{ Fault description } & \multicolumn{3}{|c|}{ Relevant measurements } \\
\hline & & Point & Measurement description & Unit \\
\hline Shaft- coupling & Loosened shaft coupling & $\mathrm{M}^{\mathrm{a}} 43$ & Vibration of the turbine guide bearing ( $X$ side) & $\mathrm{mm} / \mathrm{s}$ \\
\hline- & - & M44 & Vibration of the turbine guide bearing ( $Y$ side) & $\mathrm{mm} / \mathrm{s}$ \\
\hline Shaft- guide bearing & Too large bearing clearance & M88 & Vibration of the lower guide bearing ( $X$ side) & $\mu \mathrm{m}$ \\
\hline- & - & M89 & Vibration of the lower guide bearing ( $Y$ side) & $\mu \mathrm{m}$ \\
\hline Shaft- guide bearing & Faulty guide bearing components & M26 & Temperature of the upper guide bearing (position 1) & ${ }^{\circ} \mathrm{C}$ \\
\hline- & - & M27 & Temperature of the upper guide bearing (position 2) & ${ }^{\circ} \mathrm{C}$ \\
\hline Generator- rotor & Out-of-round rotor & M35 & Vibration of the lower guide bearing ( $X$ side) & $\mathrm{mm} / \mathrm{s}$ \\
\hline- & - & M36 & Vibration of the lower guide bearing ( $Y$ side) & $\mathrm{mm} / \mathrm{s}$ \\
\hline - & - & M40 & Vibration of the upper guide bearing ( $X$ side) & $\mathrm{mm} / \mathrm{s}$ \\
\hline- & - & M41 & Vibration of the upper guide bearing ( $Y$ side) & $\mathrm{mm} / \mathrm{s}$ \\
\hline Generator- magnetizer & Thyristor discharger malfunction & M9 & Alternator voltage & $\mathrm{kV}$ \\
\hline Generator- cooling system- control & Fault in the generator for cooling water control & M83 & Opening percentage of the fine water control valve & $\%$ \\
\hline- & - & M84 & Opening percentage of the stator control valve & $\%$ \\
\hline \multirow[t]{2}{*}{ Turbine- hydraulic pressure system } & Safety valve opens at a wrong pressure & M50 & Level of the control oil & $\mathrm{cm}$ \\
\hline & - & M51 & Level of the pressure accumulator 1 & $\mathrm{~cm}$ \\
\hline- & - & M58 & Pressure of the regulating oil & bar \\
\hline Turbine- hydraulic pressure system & Improper oil level in the piston accumulator & M51 & Level of the pressure accumulator 1 & $\mathrm{~cm}$ \\
\hline- & - & M52 & Level of the pressure accumulator 2 & $\mathrm{~cm}$ \\
\hline- & - & M53 & Level of the pressure accumulator 3 & $\mathrm{~cm}$ \\
\hline Turbine- hydraulic pressure system & Regular pump does not deliver the required capacity & M58 & Pressure of the regulating oil & bar \\
\hline
\end{tabular}

${ }^{\text {a }} \mathrm{M}$ represents measurement points.

based on historical failure data which were not successfully detected by the current method.

As expected, the algorithm yielded a satisfactory result which can be seen from Table 2. The topmost observations with the highest overall local outlier score are listed in Table 2, in which retained dimensions for each data point are also ranked by the single-dimensional local outlier scores in a descending order. The output of the algorithm shows that $90 \%$ of the faults were detected as the observations among the highest local outlier score list, and the missed fault (observation 1002) has the overall local outlier score 9.18 and was ranked at number 46 . The 512th data point may be considered as a false alarm even though it indeed deviates from other points in the retained dimension by looking into the data. 
Table 2

Topmost observations with the highest overall local outlier score

\begin{tabular}{|c|c|c|c|c|c|c|c|}
\hline \multirow[t]{2}{*}{ Rank } & \multirow[t]{2}{*}{ Observation ID } & \multirow[t]{2}{*}{ Overall local outlier score } & \multicolumn{4}{|c|}{ Feature ordering by local outlier score on each individual dimension ${ }^{\text {a }}$} & \multirow[t]{2}{*}{ Faulty or not } \\
\hline & & & Measurement 1 & Measurement 2 & Measurement 3 & Measurement 4 & \\
\hline 1 & 1009 & 710.27 & $\mathrm{M}^{\mathrm{b}} 79(592.31)^{\mathrm{c}}$ & M72 (379.57) & M54 (97.86) & & $\sqrt{ }$ \\
\hline 2 & 1004 & 642.1 & M54 (642.1) & & & & $\sqrt{ }$ \\
\hline 3 & 1008 & 641.75 & M30 (401.96) & M6 (355) & M43 (291.95) & M31 (197.5) & $\sqrt{ }$ \\
\hline 4 & 1010 & 182.32 & M74 (182.32) & & & & $\sqrt{ }$ \\
\hline 5 & 1001 & 102.42 & M23 (59.24) & M82 (59.24) & M83 (58.92) & & $\sqrt{ }$ \\
\hline 6 & 1007 & 91.4 & M88 (59.04) & M90 (55.67) & M89 (30.7) & M92 (28.76) & $\sqrt{ }$ \\
\hline 7 & 1005 & 46.34 & M43 (30.68) & M91 (25.23) & M58 (23.87) & & $\sqrt{ }$ \\
\hline 8 & 1006 & 31.97 & M43 (25.16) & M44 (19.73) & & & $\sqrt{ }$ \\
\hline 9 & 512 & 23.52 & M20 (23.52) & & & & $\times$ \\
\hline 10 & 1003 & 22.67 & M78 (16.15) & M24 (15.91) & & & $\sqrt{ }$ \\
\hline
\end{tabular}

a the retained dimensions are ranked in descending order by the local outlier score on each individual dimension;

b measurement point;

${ }^{c}$ local outlier score on each dimension is enclosed in the parenthesis.

Fault detection is commonly followed by a fault diagnosis process. A preliminary explanation for the abnormal behavior of the identified anomalous data objects in this phase can vastly assist in diagnosing the underlying fault types and sources. Although the retained subspace and ordered feature list are insufficient to directly suggest the fault type and source, it can narrow down the scope of root cause analysis greatly. For example, the fault of the observation 1007 shown in Table 2 most probably stems from the shaft of the system. The algorithm not only gives an outlier score for each observation but also sorts the retained features according to the single-dimensional local outlier score for any potential faults. In summary, feature ordering in the relevant subspace can be very informative for an ensuing fault diagnosis.

\section{Conclusions}

This paper proposes an Angle-based Subspace Anomaly Detection approach to detecting anomalies from high-dimensional datasets. The approach selects relevant subspaces from full-dimensional spaces based on the angle between all pairs of two lines for one specific anomaly candidate: the first line is connected by the concerned point and the center of its surrounding points; the second one is one of the axis-parallel lines. The angle is calculated by the metric "pairwise cosine" (PCOS). The so-called PCos is the average absolute value of cosine between the projections of the two lines on all possible twodimensional spaces. Each of these two-dimensional spaces is spanned by the concerned axis dimension and one of the remaining dimensions of the feature space. The dimensions that have a relatively large $P C o s$ value constitute the targeted subspace. For computing the local outlier-ness of the anomaly candidate in its subspace projection, a normalized Mahalanobis distance measure is used.

Based on the analytical study and numerical illustration, we can conclude that: (i) the proposed approach can retain dimensions which present a large discrepancy between points and their neighboring points, i.e. a meaningful subspace. (ii) the proposed metric "pairwise cosine" for measuring vectorial angles in high-dimensional spaces is a bounded metric and it is asymptotically stable as dimensionality increases. (iii) the experiment on synthetic datasets with various dimensionality settings indicates that the suggested algorithm can detect anomalies effectively and has a superior accuracy over the specified alternatives in high-dimensional spaces. (iv) the experiment on the industrial dataset shows the applicability of the algorithm in real-world applications, and feature ordering in relevant subspaces is informative to the ensuing analysis and diagnosis to abnormality.

The proposed algorithm can be adapted to an online mode to detect anomalies from data stream in real time, as has been done in [24]. It can also be extended to deal with nonlinear systems by introducing the kernel method [39]. The kernel method maps the raw data from input space to a Hilbert space (often with higher dimensions or even infinite), on which anomalies and normal samples may be more easily separable. We consider these extensions as future research work.

\section{Acknowledgments}

The authors would like to thank the Editor, the Associate Editors, and the referees for their constructive comments and suggestions that greatly improved the content of this paper.

\section{Appendix}

This section proves the three propositions presented in Section 3.4.1.

1. Proof to the proposition $\lim _{n \rightarrow \infty}\left(\mathrm{E}\left[\left|\cos \left(\vec{l}, \vec{\mu}_{n}(j)\right)\right|\right]\right)=0$,
which $\mathrm{E}\left[\left|\cos \left(\vec{l}, \vec{\mu}_{n}(j)\right)\right|\right]=\frac{1}{n} \cdot \frac{\left|l_{1}\right|+\left|l_{2}\right|+\ldots+\left|l_{n}\right|}{\sqrt{l_{1}^{2}+l_{2}^{2}+\ldots+l_{n}^{2}}}$, where $j \in N$

Proof:

The exceptional case when $l_{1}=l_{2}=\ldots=l_{n}=0$ has been discussed separately, so we suppose $l_{1}, l_{2}, \ldots, l_{n}$ cannot be 0 simultaneously. This is equivalent to prove that,

$$
\begin{aligned}
\lim _{n \rightarrow \infty}\left(\frac{x_{1}+x_{2}+\ldots+x_{n}}{n \sqrt{x_{1}^{2}+x_{2}^{2}+\ldots+x_{n}^{2}}}\right) & =0, \text { where } x_{1}, x_{2}, \ldots, x_{n} \\
& \geq 0 \text { and } x_{1}+x_{2}+\ldots+x_{n}>0
\end{aligned}
$$

Assume that $M=\max \left\{x_{1}, x_{2}, \ldots, x_{n}\right\}$, and $m=\min \left\{x_{1}, x_{2}, \ldots, x_{n}\right\}$, then

$n \cdot m \leq x_{1}+x_{2}+\ldots+x_{n} \leq n \cdot M$

and

$\sqrt{n} \cdot m \leq \sqrt{x_{1}^{2}+x_{2}^{2}+\ldots+x_{n}^{2}} \leq \sqrt{n} \cdot M$

Following inequality always holds true,

$\lim _{n \rightarrow \infty}\left(\frac{n \cdot m}{n \cdot \sqrt{n} \cdot M}\right) \leq \lim _{n \rightarrow \infty}\left(\frac{x_{1}+x_{2}+\ldots+x_{n}}{n \sqrt{x_{1}^{2}+x_{2}^{2}+\ldots+x_{n}^{2}}}\right) \leq \lim _{n \rightarrow \infty}\left(\frac{n \cdot M}{n \cdot \sqrt{n} \cdot m}\right)$ 
Since,

$\lim _{n \rightarrow \infty}\left(\frac{n \cdot m}{n \cdot \sqrt{n} \cdot M}\right)=\lim _{n \rightarrow \infty}\left(\frac{m}{\sqrt{n} \cdot M}\right)=0$

$\lim _{n \rightarrow \infty}\left(\frac{n \cdot M}{n \cdot \sqrt{n} \cdot m}\right)=\lim _{n \rightarrow \infty}\left(\frac{M}{\sqrt{n} \cdot m}\right)=0$

According to the Squeeze theorem,

$\lim _{n \rightarrow \infty}\left(\frac{x_{1}+x_{2}+\ldots+x_{n}}{n \sqrt{x_{1}^{2}+x_{2}^{2}+\ldots+x_{n}^{2}}}\right)=0$

And the rate of convergence is $1 / \sqrt{n}$.

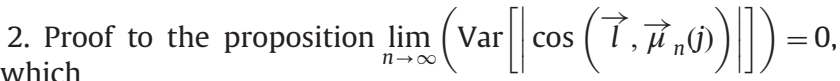

$\operatorname{Var}\left[\left|\cos \left(\vec{l}, \vec{\mu}_{n}(j)\right)\right|\right]=\frac{1}{n}-\frac{\left(\left|l_{1}\right|+\left|l_{2}\right|+\ldots+\left|l_{n}\right|\right)^{2}}{n^{2} \cdot\left(l_{1}^{2}+l_{2}^{2}+\ldots+l_{n}^{2}\right)}$, where $j \in N$

\section{Proof:}

Similarly, we suppose $l_{1}, l_{2}, \ldots, l_{n}$ cannot be 0 simultaneously. This is equivalent to proving that,

$$
\begin{aligned}
\lim _{n \rightarrow \infty}\left[\frac{1}{n}-\frac{\left(x_{1}+x_{2}+\ldots+x_{n}\right)^{2}}{n^{2}\left(x_{1}^{2}+x_{2}^{2}+\ldots+x_{n}^{2}\right)}\right] & =0 \text {, where } x_{1}, x_{2}, \ldots, x_{n} \\
& \geq 0 \text { and } x_{1}+x_{2}+\ldots+x_{n}>0
\end{aligned}
$$

Assume that $M=\max \left\{x_{1}, x_{2}, \ldots, x_{n}\right\}$, and $m=\min \left\{x_{1}, x_{2}, \ldots, x_{n}\right\}$, then

$n^{2} \cdot m^{2} \leq\left(x_{1}+x_{2}+\ldots+x_{n}\right)^{2} \leq n^{2} \cdot M^{2}$

and,

$n \cdot m^{2} \leq\left(x_{1}^{2}+x_{2}^{2}+\ldots+x_{n}^{2}\right) \leq n \cdot M^{2}$

The above two inequalities suffice the following,

$\lim _{n \rightarrow \infty}\left(\frac{1}{n}-\frac{n^{2} \cdot M^{2}}{n^{2} \cdot n \cdot m^{2}}\right) \leq \lim _{n \rightarrow \infty}\left[\frac{1}{n}-\frac{\left(x_{1}+x_{2}+\ldots+x_{n}\right)^{2}}{n^{2}\left(x_{1}^{2}+x_{2}^{2}+\ldots+x_{n}^{2}\right)}\right] \leq \lim _{n \rightarrow \infty}\left(\frac{1}{n}-\frac{n^{2} \cdot m^{2}}{n^{2} \cdot n \cdot M^{2}}\right)$

Since,

$\lim _{n \rightarrow \infty}\left(\frac{1}{n}-\frac{n^{2} \cdot M^{2}}{n^{2} \cdot n \cdot m^{2}}\right)=\lim _{n \rightarrow \infty} \frac{1}{n}\left(1-\frac{M^{2}}{m^{2}}\right)=0$

and,

$\lim _{n \rightarrow \infty}\left(\frac{1}{n}-\frac{n^{2} \cdot m^{2}}{n^{2} \cdot n \cdot M^{2}}\right)=\lim _{n \rightarrow \infty} \frac{1}{n}\left(1-\frac{m^{2}}{M^{2}}\right)=0$

According to the Squeeze theorem,

$\lim _{n \rightarrow \infty}\left[\frac{1}{n}-\frac{\left(x_{1}+x_{2}+\ldots+x_{n}\right)^{2}}{n^{2}\left(x_{1}^{2}+x_{2}^{2}+\ldots+x_{n}^{2}\right)}\right]=0$

And the rate of convergence is $1 / n$.

3. Proof to the proposition $\frac{1}{2}<\mathrm{E}\left[P \operatorname{Cos}\left(\vec{l}, \vec{\mu}_{n}(j)\right)\right] \leq \frac{\sqrt{2}}{2}$, in which

$$
\mathrm{E}\left[P \operatorname{Cos}\left(\vec{l}, \vec{\mu}_{n}(j)\right)\right]=\frac{1}{n \cdot(n-1)} \sum_{\substack{j, j^{-} \in N \\ j^{-} \neq j}} \frac{\left|l_{j}^{\#}\right|+\left|l_{j^{-}}^{\#}\right|}{\sqrt{l_{j}^{\# 2}+l_{j^{-}}^{\# 2}}},
$$

where $N=\{1,2, \ldots, n\}$, and $l_{j}^{\#}>0$ for all $j \in N$
This is equivalent to proving the following inequality,

$$
\frac{1}{2}<\frac{1}{n \cdot(n-1)} \sum_{i, j \in\{1,2, \ldots n\}} \frac{x_{i}+x_{j}}{\sqrt{x_{i}^{2}+x_{j}^{2}}} \leq \frac{\sqrt{2}}{2} \text {, where } x_{1}, x_{2}, \ldots, x_{n}>0
$$

Firstly, we prove

$1<\frac{x_{i}+x_{j}}{\sqrt{x_{i}^{2}+x_{j}^{2}}} \leq \sqrt{2}$, where $x_{i}>0, x_{j}>0$

- For the left part of the formula,

$$
\begin{aligned}
& \Leftarrow\left(x_{i}+x_{j}\right)>\sqrt{x_{i}^{2}+x_{j}^{2}} \\
& \Leftarrow\left(x_{i}^{2}+2 x_{i} x_{j}+x_{j}^{2}\right)>x_{i}^{2}+x_{j}^{2} \\
& \Leftarrow 2 x_{i} x_{j}>0
\end{aligned}
$$

Apparently, $2 x_{i} x_{j}>0$ holds true when $x_{i}>0$ and $x_{j}>0$

- For the right part of the formula,

$$
\begin{aligned}
& \Leftarrow \sqrt{2} \cdot \sqrt{x_{i}^{2}+x_{j}^{2}} \geq\left(x_{i}+x_{j}\right) \\
& \Leftarrow 2 \cdot\left(x_{i}^{2}+x_{j}^{2}\right) \geq\left(x_{i}+x_{j}\right)^{2} \\
& \Leftarrow\left(x_{i}^{2}-2 x_{i} x_{j}+x_{j}^{2}\right) \geq 0 \\
& \Leftarrow\left(x_{i}-x_{j}\right)^{2} \geq 0
\end{aligned}
$$

Apparently, $\left(x_{i}-x_{j}\right)^{2} \geq 0$ always holds true.

Now, we prove the original proposition. Since there are $n \cdot(n-1) / 2$ elements in the summation notation of the original formula and all of the elements have the form of $\left(x_{i}+x_{j}\right) / \sqrt{x_{i}^{2}+x_{j}^{2}}$ and $x_{i}>0, x_{j}>0$.

- if all the elements take the maximum value $\sqrt{2}$, i.e. $x_{1}=x_{2}=\ldots=x_{n}$, then

$$
\frac{1}{n \cdot(n-1)} \sum_{\substack{i, j \in\{1,2, \ldots n\} \\ i \neq j}} \frac{x_{i}+x_{j}}{\sqrt{x_{i}^{2}+x_{j}^{2}}}=\frac{1}{n \cdot(n-1)} \cdot \frac{n \cdot(n-1)}{2} \cdot \sqrt{2}=\frac{\sqrt{2}}{2}
$$

So the right part is proved, i.e.

$$
\frac{1}{n \cdot(n-1)} \sum_{\substack{i, j \in\{1,2, \ldots n\} \\ i \neq j}} \frac{x_{i}+x_{j}}{\sqrt{x_{i}^{2}+x_{j}^{2}}} \leq \frac{\sqrt{2}}{2}
$$

- Again since all the elements are greater than 1 , then

$$
\frac{1}{n \cdot(n-1)} \sum_{\substack{i, j \in\{1,2, \ldots n\} \\ i \neq j}} \frac{x_{i}+x_{j}}{\sqrt{x_{i}^{2}+x_{j}^{2}}}>\frac{1}{n \cdot(n-1)} \cdot \frac{n \cdot(n-1)}{2} \cdot 1=\frac{1}{2}
$$


So the left part is proved, i.e.

$$
\frac{1}{n \cdot(n-1)} \sum_{\substack{i, j \in\{1,2, \ldots n\} \\ i \neq j}} \frac{x_{i}+x_{j}}{\sqrt{x_{i}^{2}+x_{j}^{2}}}>\frac{1}{2}
$$

Thus, the original proposition is right.

\section{References}

[1] Montgomery DC. Big data and the quality profession. Qual Reliab Eng Int 2014;30(4):447.

[2] Sribar VT, Feinberg D, Gall N, Lapkin A, Beyer MA., Big Data is only the beginning of extreme information management. Gartner, Stamford, CT; 2011

[3] Zhai Y, Ong Y-S, Tsang IW. The emerging big dimensionality. Comput Intell Mag IEEE 2014;9(3):14-26.

[4] Meeker WQ, Hong Y. Reliability meets big data: opportunities and challenges. Qual Eng 2014;26(1):102-16.

[5] Domingos P. A few useful things to know about machine learning. Commun ACM 2012;55(10):78-87.

[6] Göb R. Discussion of reliability meets big data: opportunities and challenges. Qual Eng 2013;26(1):121-6.

[7] Hawkins DM. Identification of outliers. 1980.

[8] Guo B, Wang BX, Xie M. A study of process monitoring based on inverse Gaussian distribution. Comput Ind Eng 2014;76:49-59.

[9] Baraldi P, Roozbeh R-F, Zio E. Classifier-ensemble incremental-learning procedure for nuclear transient identification at different operational conditions. Reliab Eng Syst Saf 2011;96(4):480-8.

[10] Albaghdadi M, Briley B, Evens M. Event storm detection and identification in communication systems. Reliab Eng Syst Saf 2006;91(5):602-13.

[11] Houle ME, Kriegel H-P, Kröger P, Schubert E, Zimek A. Can shared-neighbor distances defeat the curse of dimensionality? Scientific and Statistical Database Management. Berlin Heidelberg: Springer; 2010. p. 482-500.

[12] Zimek A, Schubert E, Kriegel H-P. A survey on unsupervised outlier detection in high-dimensional numerical data,. Stat Anal Data Min 2012;5(5):363-87.

[13] Dai X, Gao Z. From model, signal to knowledge: a data-driven perspective of fault detection and diagnosis. IEEE Trans Ind Inf 2013;9(4):2226-38.

[14] Zhong S, Langseth H, Nielsen TD. A classification-based approach to monitoring the safety of dynamic systems,. Reliab Eng Syst Saf 2014;121:61-71.

[15] Rocco S. CM, Zio E. A support vector machine integrated system for the classification of operation anomalies in nuclear components and systems. Reliab Eng Syst Saf 2007;92(5):593-600.

[16] Tamilselvan P, Wang P. Failure diagnosis using deep belief learning based health state classification. Reliab Eng Syst Saf 2013;115:124-35.

[17] Traore M, Chammas A, Duviella E. Supervision and prognosis architecture based on dynamical classification method for the predictive maintenance of dynamical evolving systems. Reliab Eng Syst Saf 2015;136:120-31.
[18] Li F, Church G, Janakiram M, Gholston H, Runger G. Fault detection for batch monitoring and discrete wavelet transforms,. Qual Reliab Eng Int 2011;27 (8):999-1008.

[19] Huang S-P, Chiu C-C. Process monitoring with ICA-based signal extraction technique and CART approach. Qual Reliab Eng Int 2009;25(5):631-43.

[20] Hwang W-Y, Lee J-S. Shifting artificial data to detect system failures. Int Trans Oper Res 2015;22(2):363-78.

[21] Lee J-M, Qin SJ, Lee I-B. Fault detection and diagnosis based on modified independent component analysis. AIChE J 2006:52(10):3501-14.

[22] Lee J, Kang B, Kang S-H. Integrating independent component analysis and local outlier factor for plant-wide process monitoring. J Process Control 2011;21(7):1011-21.

[23] Zhao Y, Wang S, Xiao F. Pattern recognition-based chillers fault detection method using support vector data description (SVDD). Appl Energy 2013; $112: 1041-8$

[24] Ma Y, Shi H, Ma H, Wang M. Dynamic process monitoring using adaptive local outlier factor. Chemom Intell Lab Syst 2013;127:89-101.

[25] Chandola V, Banerjee A, Kumar V. Anomaly detection: a survey. ACM Comput Surv 2009;41(3):1-72.

[26] Li J, Huang K-Y, Jin J, Shi J. A survey on statistical methods for health care fraud detection. Health Care Manag Sci 2008;11(3):275-87.

[27] Knorr EM, Ng RT, Tucakov V. Distance-based outliers: algorithms and applications,. VLDB J Int J Very Large Data Bases 2000;8(3-4):237-53.

[28] Kriegel H-P, Zimek A. Angle-based outlier detection in high-dimensional data. In: Proceedings of the 14th ACM SIGKDD international conference on Knowledge discovery and data mining; 2008. p. 444-52.

[29] Breunig MM, Kriegel H, Ng RT, Sander J. LOF : identifying density-based local outliers. ACM Sigmod Rec 2000;29(2):93-104.

[30] Beyer K, Goldstein J, Ramakrishnan R, Shaft U. When is 'nearest neighbor' meaningful? Database Theory-ICDT'99. Berlin Heidelberg: Springer; 1999. p. 217-235.

[31] Piao C, Huang Z, Su L, Lu S. Research on outlier detection algorithm for evaluation of battery system safety. Adv Mech Eng 2014;2014:1-8.

[32] Aggarwal CC, Yu PS. Outlier detection for high dimensional data. ACM SIGMOD Rec 2001;30(2):37-46.

[33] Lazarevic A, Kumar V. Feature bagging for outlier detection. In: Proceeding of the eleventh ACM SIGKDD international conference on knowledge discovery in data mining; 2005. p. 157-66.

[34] Kriegel H, Kröger P, Schubert E, Zimek A. Outlier detection in axis-parallel subspaces of high dimensional data. Advances in Knowledge Discovery and Data Mining. Berlin Heidelberg: Springer; 2009. p. 831-8.

[35] Ye M, Li X, Orlowska ME. Projected outlier detection in high-dimensional mixed-attributes data set. Expert Syst Appl 2009;36(3):7104-13.

[36] Ayad H, Kamel M. Finding natural clusters using multi-clusterer combiner based on shared nearest neighbors. Multiple classifier systems. Berlin Heidelberg: Springer; 2003. p. 166-75.

[37] Cai T, Fan J, Jiang T. Distributions of angles in random packing on spheres. J Mach Learn Res 2013;14(1):1837-64.

[38] Song X, Wu M, Jermaine C, Ranka S. Conditional anomaly detection. IEEE Trans Knowl Data Eng 2007;19(5):631-45.

[39] Zhang Y, Qin SJ. Improved nonlinear fault detection technique and statistical analysis. AIChE J 2008;54(12):3207-20. 